\title{
Operators in the Cowen-Douglas class and related topics
}

\author{
Gadadhar Misra
}

Linear spaces with an Euclidean metric are ubiquitous in mathematics, arising both from quadratic forms and inner products. Operators on such spaces also occur naturally. In recent years, the study of multivariate operator theory has made substantial progress. Although, the study of self adjoint operators goes back a few decades, the non-self adjoint theory has developed at a slower pace. While several approaches to this topic has been developed, the one that has been most fruitful is clearly the study of Hilbert spaces that are modules over natural function algebras like $\mathscr{A}(\Omega)$, where $\Omega \subseteq \mathbb{C}^{m}$ is a bounded domain, consisting of complex valued functions which are holomorphic on some open set $U$ containing $\bar{\Omega}$, the closure of $\Omega$. The book [29] showed how to recast many of the familiar theorems of operator theory in the language of Hilbert modules. The books [31] and [14] provide an account of the achievements from the recent past. The impetus for much of what is described below comes from the interplay of operator theory with other areas of mathematics like complex geometry and representation theory of locally compact groups.

\section{INTRODUCTION}

The first half of this expository article describe several elementary properties of the operators in the Cowen-Douglas class. This is divided into five separate themes. In the second half of the article, we elaborate a little more on each of these themes.

1.1. Operators in the Cowen-Douglas class. In the paper [17], Cowen and Douglas initiated a systematic study of a class of bounded linear operators on a complex separable Hilbert space possessing an open set of eigenvalues of constant (and finite) multiplicity. Let $\Omega$ be the set of eigenvalues of the operator $T: \mathscr{H} \rightarrow \not{H}$ in this class. Assuming that $\operatorname{ran}(T-w I)=\mathscr{H}$, using elementary Fredholm theory, they prove: For a fixed but arbitrary $w_{0} \in \Omega$, there is an open neighbourhood $U$ of $w_{0}$ and holomorphic functions

$$
\gamma_{i}: U \rightarrow \not{H},(T-w) \gamma_{i}(w)=0,1 \leq i \leq n,
$$

such that the vectors $\left\{\gamma_{1}(w), \ldots, \gamma_{n}(w)\right\}$ are linearly independent, $w \in U$. They also show that such an operator $T$ defines a holomorphic Hermitian vector bundle $E_{T}$ :

$$
t: \Omega \rightarrow \operatorname{Gr}(n, \mathscr{H}), t(w)=\operatorname{ker}(T-w) \subseteq \mathscr{H} .
$$

This means, for any fixed but arbitrary point $w_{0} \in \Omega$, there exists a holomorphic map $\boldsymbol{\gamma}_{T}$ of the form $\boldsymbol{\gamma}_{T}(w)=$ $\left(\gamma_{1}(w), \ldots, \gamma_{n}(w)\right),(T-w) \gamma_{i}(w)=0$ in some open neighbourhood $U$ of $w_{0}$. It is called a holomorphic frame for the operator $T$. Finally, Cowen and Douglas also assume that the linear span of $\left\{\gamma_{1}(w), \ldots, \gamma_{n}(w): w \in \Omega\right\}$ is dense in $\mathscr{H}$. Let $B_{n}(\Omega)$ denote this class of operators.

One of the striking results of Cowen and Douglas says that there is a one to one correspondence between the unitary equivalence class of the operators $T$ and the (local) equivalence classes of the holomorphic Hermitian vector bundles $E_{T}$ determined by them. As a result of this correspondence set up by the CowenDouglas theorem, the invariants the vector bundle $E_{T}$ like the curvature, the second fundamental form, etc.

Key words and phrases. Cowen-Douglas class, Curvature inequalities, Homogeneous operators and vector bundles, Hilbert Module, Quotient and Sub modules, Flag structure.

The author gratefully acknowledges the financial support from the Department of Science and Technology in the form of the J C Bose National Fellowship and from the University Grants Commission, Centre for Advanced Study. 
now serve as unitary invariants for the operator $T$. Although finding a complete set of tractable invariants, not surprisingly, is much more challenging. Examples were given in [51, Example 2.1] to show that the class of the curvature alone does not determine the class of the vector bundle except in the case of a line bundle. Before we consider this case in some detail, let us recall the interesting notion of a spanning section. A holomorphic function $s: \Omega \rightarrow \mathscr{H}$ is called a spanning section for an operator $T$ in the Cowen-Douglas class if $\operatorname{ker}(T-w) s(w)=0$ and the closed linear span of $\{s(w): w \in \Omega\}$ is $\mathscr{H}$. Kehe Zhu in [62] proved the existence of a spanning section for an operator $T$ in $B_{n}(\Omega)$ and showed that it can be used to characterize Cowen-Douglas operators of rank $n$ up to unitary equivalence and similarity. Unfortunately, the existential nature of the spanning section makes it difficult to apply this result in concrete examples.

First note that the holomorphic frame $\gamma_{T}$ is not uniquely determined even if the rank $n=1$. If $\gamma_{T}$ is any given holomorphic frame for the operator $T$ defined on an open set $\Omega \subseteq \mathbb{C}$ and $\varphi: \Omega \rightarrow \mathbb{C}$ is a nonvanishing holomorphic function, then $\varphi \gamma_{T}$ is also a holomorphic frame for the line bundle $E_{T}$. Therefore, a holomorphic frame can't possibly determine the unitary equivalence class of the operator $T$. How does one get rid of this ambiguity in the holomorphic frame to obtain an invariant? It is evident that

$$
\mathscr{K}_{T}(w)=-\frac{\partial^{2}}{\partial w \partial \bar{w}} \log \left\|\gamma_{T}(w)\right\|^{2}, w \in \Omega_{0},
$$

is the same for all holomorphic frames of the form $\varphi \gamma_{T}$, where $\varphi$ is any non-vanishing holomorphic function on some open set $\Omega_{0} \subseteq \Omega$. Since any two holomorphic frames of the operator $T$ must differ by such a holomorphic change of frame, we conclude that $\mathscr{K}_{T}$ is a unitary invariant for the operator $T$. The converse is also valid and is well-known: The curvature $\mathscr{K}_{T}$ of the line bundle $E_{T}$ is defined by the formula (1.1) and is a complete invariant for the line bundle $E_{T}$.

To see the usefulness of this invariant, consider the weighted unilateral shift $W_{\lambda}$ determined by the weight sequence $\left\{\sqrt{\frac{n+1}{n+\lambda}}\right\}, \lambda>0$, acting on the Hilbert space $\ell^{2}$ of square summable sequences. Clearly, the adjoint $W_{\lambda}^{*}$ admits a holomorphic frame. For instance, one may choose $\gamma_{W_{\lambda}^{*}}(w)=\left(1, c_{1} w, c_{2} w^{2}, \ldots\right), w \in \mathbb{D}$, where $c_{k}^{2}$ is the co-efficient of $x^{k}$ in the binomial expansion of the function $(1-x)^{-\lambda}$. It then follows that $\left\|\gamma_{W_{\lambda}^{*}}(w)\right\|^{2}=\left(1-|w|^{2}\right)^{-\lambda}$ and that $\mathscr{K}_{W_{\lambda}^{*}}(w)=-\lambda\left(1-|w|^{2}\right)^{-2}, w \in \mathbb{D}$. Consequently, using the CowenDouglas theorem, we conclude that none of the operators $W_{\lambda}$ are unitarily equivalent among themselves.

Finding similarity invariants for operators in the class $B_{n}(\Omega)$ has been somewhat difficult from the beginning. The conjecture made by Cowen and Douglas in [17] was shown to be false [15, 16]. However, significant progress on the question of similarity has been made recently (cf. [36, 37, 23, 40]).

After a model and a complete set of unitary invariants were provided for the operators in the class $B_{n}(\Omega)$ in [17], it was only natural to follow it up with the study of a commuting tuple of operators with similar properties. This was started in [18] and followed up in the papers [20] and [21]. The approach in the papers [20] and [21] are quite different. We recall below the definition of the class $B_{n}(\Omega), \Omega \subset \mathbb{C}^{m}$, from the paper [21]. This definition also appears in [18] and is implicit in [20].

Let $\boldsymbol{T}=\left(T_{1}, \ldots, T_{m}\right)$ be an $m$-tuple of commuting bounded linear operators on a separable complex Hilbert space $\mathscr{H}$. For $w=\left(w_{1}, \ldots, w_{m}\right)$ in $\Omega$, let $T-w$ denote the $m$-tuple $\left(T_{1}-w_{1}, \ldots, T_{m}-w_{m}\right)$. Define the operator $D_{T}: \not{H} \rightarrow \not{H} \oplus \cdots \oplus \mathscr{H}$ by

$$
D_{T}(x)=\left(T_{1} x, \ldots, T_{m} x\right), x \in \mathscr{H} .
$$

Definition 1.1. For a connected, bounded and open subset $\Omega$ of $\mathbb{C}^{m}$, a $m$-tuple $\boldsymbol{T}$ is said to be in the CowenDouglas class $B_{n}(\Omega)$ of rank $n, n \in \mathbb{N}$, if

(1) $\operatorname{ran} D_{T-w}$ is closed for all $w \in \Omega$

(2) $\operatorname{span}\left\{\operatorname{ker} D_{T-\boldsymbol{w}}: w \in \Omega\right\}$ is dense in $\mathscr{H}$

(3) $\operatorname{dim} \operatorname{ker} D_{T-\boldsymbol{w}}=n$ for all $w \in \Omega$. 
For $m=1$, it is shown in [17, Proposition 1.12] that if $T$ is in $B_{n}(\Omega)$, then there exists a choice of $n$ eigenvectors in $\operatorname{ker}(T-w)$, which are holomorphic as functions of $w \in \Omega$ making

$$
E_{T}:=\{(w, x): w \in \Omega, x \in \operatorname{ker}(T-w)\} \subseteq \Omega \times \mathscr{H},
$$

$\pi: E_{T} \rightarrow \Omega, \pi(w, x)=w$, is a rank $n$ holomorphic Hermitian vector bundle over $\Omega$. Here is one of the main results from [17].

Theorem (Cowen and Douglas). The operators $T$ and $\hat{T}$ in $B_{n}(\Omega)$ are unitarily equivalent if and only if the corresponding holomorphic Hermitian vector bundles $E_{T}$ and $E_{\hat{T}}$ are equivalent on some open subset $\Omega_{0}$ of $\Omega$.

The existence of the vector bundle $E_{\boldsymbol{T}}$ follows from [21, Theorem 2], while [21, Theorem 3.7] provides the analogue of the Cowen-Douglas Theorem for an arbitrary $m$. Finally, a complete set of invariants in this case are given in [20].

Crucial in any study of such a class is the problem of finding a canonical model and a set of invariants. For normal operators, the spectral theorem provides a model in the form of a multiplication operator and a complete set of invariants is given by the spectrum, the spectral measure and the multiplicity function. Similarly, the Sz.-Nagy - Foias theory provides a model for a pure completely nonunitary contraction and the characteristic function serves as a complete invariant. Now, we describe a model for the operators, resp. commuting tuples, in the Cowen-Douglas class.

Let $V$ be a $n$-dimensional Hilbert space and $\mathscr{L}(V)$ denote the vector space of all linear transformations on $V$. A function $K: \Omega \times \Omega \rightarrow \mathscr{L}(V)$, satisfying

$$
\sum_{i, j=1}^{N}\left\langle K\left(w_{i}, w_{j}\right) \zeta_{j}, \zeta_{i}\right\rangle_{V} \geq 0, w_{1}, \ldots, w_{N} \in \Omega, \zeta_{1}, \ldots, \zeta_{N} \in V, N \geq 1
$$

is said to be a non negative definite (nnd) kernel on $\Omega$. Given such an nnd kernel $K$ on $\Omega$, it is easy to construct a Hilbert space $\mathscr{H}$ of functions on $\Omega$ taking values in $V$ with the property

$$
\langle f(w), \zeta\rangle_{V}=\langle f, K(\cdot, w) \zeta\rangle_{\mathscr{H}}, w \in \Omega, \zeta \in V, f \in \mathscr{H} .
$$

The Hilbert space $\mathscr{H}$ is simply the completion of the linear span of all vectors of the form $K(\cdot, w) \zeta, w \in \Omega$, $\zeta \in V$, with inner product defined by (1.3).

Conversely, let $\mathscr{H}$ be any Hilbert space of functions on $\Omega$ taking values in $V$. Let $e_{w}: \mathscr{H} \rightarrow V$ be the evaluation functional defined by $e_{w}(f)=f(w), w \in \Omega, f \in \mathscr{H}$. If $e_{w}$ is bounded for each $w \in \Omega$ then it is easy to verify that the Hilbert space $\mathscr{H}$ possesses a reproducing kernel $K(z, w)=e_{z} e_{w}^{*}$, that is, $K(\cdot, w) \zeta \in \mathscr{H}$ for each $w \in \Omega$ and $K$ has the reproducing property (1.3). Finally, the reproducing property (1.3) determines the kernel $K$ uniquely. We let $(\mathscr{H}, K)$ be the Hilbert space $\mathscr{H}$ equipped with the reproducing kernel $K$.

Remark 1.1. Let $K: \Omega \times \Omega \rightarrow \mathscr{M}_{k}(\mathbb{C})$ be a non-negative definite kernel. For every $\boldsymbol{i} \in \mathbb{Z}_{+}^{m}, \eta \in \mathbb{C}^{k}$ and $w \in \Omega$, we have

(i) $\bar{\partial}^{i} K(\cdot, w) \eta$ is in $(\mathscr{H}, K)$,

(ii) $\left\langle f, \bar{\partial}^{i} K(\cdot, w) \eta\right\rangle=\left\langle\left(\partial^{i} f\right)(w), \eta\right\rangle_{\mathbb{C}^{k}}, f \in(\mathscr{H}, K)$.

The proof follows from the uniform boundedness principle [33, Proposition 2.1.3].

Given any $m$-tuple of operators $\boldsymbol{T}$ in $B_{n}(\Omega)$, there exists an open subset $U$ of $\Omega$ and $n$ linearly independent vectors $\gamma_{1}(w), \ldots, \gamma_{n}(w)$ in $\operatorname{ker} D_{T-w}, w \in U$, such that each of the maps $w \mapsto \gamma_{i}(w)$ is holomorphic on $U$, see [17, Proposition 1.11] and [21, Theorem 2.2]. Define $\hat{\Gamma}: U \rightarrow \mathscr{L}\left(\mathbb{C}^{n}, \mathscr{H}\right)$ by setting

$$
\hat{\Gamma}(w) \zeta=\sum_{i=1}^{n} \zeta_{i} \gamma_{i}(w), \zeta=\left(\zeta_{1}, \ldots, \zeta_{n}\right) \in \mathbb{C}^{n}
$$


Let $\mathscr{O}\left(U, \mathbb{C}^{n}\right)$ denote the linear space of holomorphic functions on $U$ taking values in $\mathbb{C}^{n}$. Set $U^{*}:=\{w$ : $\bar{w} \in U\}$. Define $\Gamma: \not{H} \rightarrow \mathscr{O}\left(U^{*}, \mathbb{C}^{n}\right)$ by

$$
(\Gamma x)(w)=\hat{\Gamma}(\bar{w})^{*} x, x \in \mathscr{H}, w \in U^{*} .
$$

Define a sesqui-linear form on $\mathscr{H}_{\Gamma}=\operatorname{ran} \Gamma$ by $\langle\Gamma f, \Gamma g\rangle_{\Gamma}=\langle f, g\rangle, f, g \in \mathscr{H}$. The map $\Gamma$ is linear and injective. Hence $\mathscr{\ell}_{\Gamma}$ is a Hilbert space of $\mathbb{C}^{n}$-valued holomorphic functions on $U^{*}$ with inner product $\langle\cdot, \cdot\rangle_{\Gamma}$ and $\Gamma$ is unitary. Then it is easy to verify the following (cf. [17, pp. 194 ] and [21, Remarks 2.6]).

a): $K(z, w)=\hat{\Gamma}(\bar{z})^{*} \hat{\Gamma}(\bar{w}), z, w \in U^{*}$ is the reproducing kernel for the Hilbert space $\mathscr{H}_{\Gamma}$.

b): $M_{i}^{*} \Gamma=\Gamma T_{i}$, where $\left(M_{i} f\right)(z)=z_{i} f(z), z \in U^{*}$.

Thus any commuting $m$-tuple $\boldsymbol{T}$ of operators in the class $B_{n}(\Omega)$ may be realized as the adjoint of the $m$-tuple $\boldsymbol{M}:=\left(M_{1}, \ldots, M_{m}\right)$ of multiplication by the coordinate functions on some Hilbert space $\mathscr{H}$ of holomorphic functions defined on $U^{*}$ possessing a reproducing kernel $K$. In this representation, clearly, $\Gamma\left(\gamma_{i}(w)\right)=$ $K(\cdot, \bar{w}) \varepsilon_{i}, 1 \leq i \leq n$ is a holomorphic frame.

We give this correspondence for commuting tuple of operators in $B_{1}(\Omega)$ adding that except for slight increase in the notational complexity, the same proof works in general.

Let $\gamma$ be a non-zero holomorphic section defined on some open subset $U$ of $\Omega$ for the operator $T$ acting on the Hilbert space $\mathscr{H}$. Consider the map $\Gamma: \mathscr{H} \rightarrow \mathscr{O}\left(U^{*}\right)$ defined by $\Gamma(x)(z)=\langle x, \gamma(\bar{z})\rangle, z \in U^{*}$. Transplant the inner product from $\mathscr{H}$ on the range of $\Gamma$. The map $\Gamma$ is now unitary from $\mathscr{H}$ onto ran $\Gamma$. Define $K$ to be the function $K(z, w)=\Gamma(\gamma(\bar{w}))(z)=\langle\gamma(\bar{w}), \gamma(\bar{z})\rangle, z, w \in U^{*}$. Set $K_{w}(\cdot):=K(\cdot, w)$. Thus $K_{w}$ is the function $\Gamma(\gamma(\bar{w}))$. It is then easily verified that $K$ has the reproducing property, that is,

$$
\begin{aligned}
\langle\Gamma(x)(z), K(z, w)\rangle_{\mathrm{ran} \Gamma} & =\left\langle(\langle x, \gamma(\bar{z})\rangle),(\langle\gamma(\bar{w}), \gamma(\bar{z}))\rangle_{\mathrm{ran} \Gamma}\right. \\
& =\langle\Gamma x, \Gamma(\gamma(\bar{w}))\rangle_{\mathrm{ran} \Gamma}=\langle x, \gamma(\bar{w})\rangle_{\mathscr{H}} \\
& =\Gamma(x)(w), x \in \mathscr{H}, w \in U^{*}
\end{aligned}
$$

It follows that $\left\|K_{w}(\cdot)\right\|^{2}=K(w, w), w \in U^{*}$. Also, $K_{w}(\cdot)$ is an eigenvector for the operator $\Gamma T_{i} \Gamma^{*}, 1 \leq i \leq m$, with eigenvalue $\bar{w}_{i}$ :

$$
\begin{aligned}
\Gamma T_{i} \Gamma^{*}\left(K_{w}(\cdot)\right) & =\Gamma T_{i} \Gamma^{*}(\Gamma(\gamma(\bar{w}))) \\
& =\Gamma T_{i} \gamma(\bar{w}) \\
& =\Gamma \bar{w}_{i} \gamma(\bar{w}) \\
& =\bar{w}_{i} K_{w}(\cdot), w \in U^{*}
\end{aligned}
$$

Since the linear span of the vectors $\left\{K_{w}: w \in U^{*}\right\}$ is dense in $(\mathcal{H}, K)$ (see [17, Corollary 1.13]), it follows that $\Gamma T_{i} \Gamma^{*}$ is the adjoint $M_{i}^{*}$ of the multiplication operator $M_{i}$ acting on $(\mathscr{H}, K)$. We therefore assume, without loss of generality, that an operator $\boldsymbol{T}$ in $B_{1}(\Omega)$ has been realized as the adjoint $\boldsymbol{M}^{*}$ of the multiplication operator $\boldsymbol{M}$ on some Hilbert space $(\mathscr{H}, K)$ of holomorphic functions on $U^{*}$ possessing a reproducing kernel $K$.

Moreover, starting from any nnd kernel $K$ defined on $\Omega$ taking values in $\mathscr{M}_{n}(\mathbb{C})$ and fixing a $w_{0}$ in $\Omega$, we note that the function

$$
K_{0}(z, w)=K\left(w_{0}, w_{0}\right)^{\frac{1}{2}} \varphi(z)^{-1} K(z, w) \overline{\varphi(w)^{-1}} K\left(w_{0}, w_{0}\right)^{\frac{1}{2}}
$$

is defined on some open neighbourhood $U$ of $w_{0}$ on which $\varphi(z)=K\left(z, w_{0}\right)$ is holomorphic and non-zero. Thus, the $m$ - tuple $\boldsymbol{M}$ defined on $(\mathscr{H}, K)$ is unitarily equivalent to the the $m$ - tuple $\boldsymbol{M}$ on $\left(\mathscr{H}_{0}, K_{0}\right)$, see [17, 21].

The kernel $K_{0}$ is said to be normalized at $w_{0}$ in the sense that $K_{0}\left(z, w_{0}\right)=I_{n}$ for each $z \in U$. 
The commuting $m$-tuple of multiplication operators acting on the Hilbert space $\mathscr{H}_{\Gamma}$ is called the canonical model. This terminology is justified by [21, Theorem 4.12(a)], it says, "the canonical models associated with two generalized Bergman kernels are unitarily equivalent if and only if the normalized forms of the kernels are unitarily equivalent via a unitary that does not depend on points of $\Omega$."

It is possible to impose conditions on a kernel function $K: \Omega \times \Omega \rightarrow \mathbb{C}$ so that each of the multiplication operators $M_{1}, \ldots, M_{m}$ are bounded on the Hilbert space $(\mathscr{H}, K)$. Additional conditions, explicitly given in [21], on $K$ ensure that $\boldsymbol{M}^{*}:=\left(M_{1}^{*}, \ldots, M_{m}^{*}\right)$ is in $B_{1}\left(\Omega^{*}\right)$. If we set the curvature $\mathscr{K}$ of the $m$-tuple $\boldsymbol{M}^{*}$ to be the $(1,1)$ - form

$$
\mathscr{K}(w):=-\sum_{i, j=1}^{m} \mathscr{K}_{i, j}(w) d w_{i} \wedge d \bar{w}_{j}
$$

where $\mathscr{K}_{i, j}(\bar{w})=\left(\frac{\partial^{2}}{\partial w_{i} \partial \bar{w}_{j}} \log K\right)(w, w)$, then the unitary equivalence class of operators $\boldsymbol{T}$ in $B_{1}\left(\Omega^{*}\right)$, which we assume is of the form $\boldsymbol{M}^{*}$ on some reproducing kernel Hilbert space $(\mathscr{H}, K)$, is determined by the curvature $(1,1)$ form.

In the case of a commuting $m$-tuple of operators $\boldsymbol{T}$ in the Cowen-Douglas class $B_{n}(\Omega)$, the existence of a spanning section was proved in [32]. Some examples of spanning sections are given in [8].

1.2. Curvature inequalities. We may assume, without loss of generality, that an operator $T$ in $B_{1}(\Omega)$ has been realized as the adjoint $M^{*}$ of the multiplication operator $M$ on some Hilbert space $(\mathcal{H}, K)$ of holomorphic functions on $\Omega^{*}$ possessing a reproducing kernel $K: \Omega^{*} \times \Omega^{*} \rightarrow \mathbb{C}$. For the unit disc $\mathbb{D}$, the distinction between $\mathbb{D}$ and $\mathbb{D}^{*}$ disappears and we write $K(z, w)$, when strictly speaking, we should be writing $K(\bar{z}, \bar{w}), z, w \in \mathbb{D}$. The curvature of the operator $M^{*}$ may be also written in the form

$$
\mathscr{K}(w)=-\frac{\|\gamma(w)\|^{2}\left\|\gamma^{\prime}(w)\right\|^{2}-\left|\left\langle\gamma^{\prime}(w), \gamma(w)\right\rangle\right|^{2}}{\|\gamma(w)\|^{4}}
$$

for some holomorphic frame $\gamma$. In particular, choosing $\gamma(\bar{w})=K(\cdot, w), w \in \mathbb{D}$, we also have

$$
\mathscr{K}(\bar{w})=-\frac{\partial^{2}}{\partial w \partial \bar{w}} \log K(w, w)=-\frac{K(w, w)(\partial \bar{\partial} K)(w, w)-|(\partial K)(w, w)|^{2}}{K(w, w)^{2}} .
$$

In either case, since $K$ is nnd, the Cauchy - Schwarz inequality applies, and we see that the numerator is non-negative. Therefore, $\frac{\partial^{2}}{\partial w \partial \bar{w}} \log K(w, w)$ must be a non-negative function.

The contractivity of the adjoint $M^{*}$ of the multiplication operator $M$ on some reproducing kernel Hilbert space $(\mathscr{H}, K)$ is equivalent to the requirement that $K^{\ddagger}(z, w):=(1-z \bar{w}) K(z, w)$ is nnd on $\mathbb{D}$. This is easy to prove as long as $K$ is positive definite. However, with a little more care, one can show this assuming only that $K$ is nnd, see [33, Lemma 2.1.10].

Now, let $T$ be any contraction in $B_{1}(\mathbb{D})$ realized in the form of the adjoint $M^{*}$ of the multiplication operator $M$ on some reproducing kernel Hilbert space $(\mathscr{H}, K)$. Then we have

$$
\frac{\partial^{2}}{\partial w \partial \bar{w}} \log K(w, w)=\frac{\partial^{2}}{\partial w \partial \bar{w}} \log \frac{1}{\left(1-|w|^{2}\right)}+\frac{\partial^{2}}{\partial w \partial \bar{w}} \log K^{\ddagger}(w, w), w \in \mathbb{D} .
$$

Let $S$ be the unilateral shift acting on $\ell^{2}$. Choosing a holomorphic frame $\gamma_{S^{*}}$, say $\gamma_{S^{*}}(w)=\left(1, w, w^{2}, \ldots\right)$, it follows that $\left\|\gamma_{S^{*}}(w)\right\|^{2}=\left(1-|w|^{2}\right)^{-1}$ and that $\mathscr{K}_{S^{*}}(w)=-\left(1-|w|^{2}\right)^{-2}, w \in \mathbb{D}$. We can therefore, rewrite the previous equality in the form

$$
\mathscr{K}_{M^{*}}(w)-\mathscr{K}_{S^{*}}(w)=-\frac{\partial^{2}}{\partial w \partial \bar{w}} \log K^{\ddagger}(w, w) \leq 0, w \in \mathbb{D} .
$$

In consequence, we have

$$
\mathscr{K}_{M^{*}}(w) \leq \mathscr{K}_{S^{*}}(w), w \in \mathbb{D}
$$


Thus the the operator $S^{*}$ is an extremal operator in the class of all contractive Cowen-Douglas operator in $B_{1}(\mathbb{D})$. The extremal property of the operator $S^{*}$ prompts the following question due to R. G. Douglas.

A question of $R$. G. Douglas. For a contraction $T$ in $B_{1}(\mathbb{D})$, if $\mathscr{K}_{T}\left(w_{0}\right)=-\left(1-\left|w_{0}\right|^{2}\right)^{-2}$ for some fixed $w_{0}$ in $\mathbb{D}$, then does it follow that $T$ must be unitarily equivalent to the operator $S^{*}$ ?

It is known that the answer is negative, in general, however it has an affirmative answer if, for instance, $T$ is a homogeneous contraction in $B_{1}(\mathbb{D})$, see [47]. From the simple observation that $\mathscr{K}_{T}(\bar{\zeta})=-\left(1-|\zeta|^{2}\right)^{-2}$ for some $\zeta \in \mathbb{D}$ if and only if the two vectors $K_{\zeta}^{\ddagger}$ and $\bar{\partial} K_{\zeta}^{\ddagger}$ are linearly dependent, it follows that the question of Douglas has an affirmative answer in the class of contractive, co-hyponormal backward weighted shifts. The Question of Douglas for all those operators $T$ in $B_{1}(\mathbb{D})$ possessing two additional properties, namely, $T^{*}$ is 2 hyper-contractive and $(\phi(T))^{*}$ has the wandering subspace property for any bi-holomorphic automorphism $\phi$ of $\mathbb{D}$ mapping $\zeta$ to 0 . This is Theorem 3.6 of the of the paper [50].

Now suppose that the domain $\Omega$ is not simply connected. In this case, replacing the contractivity of the operator $T$ by the contractivity of the homomorphism $\varrho_{T}$ induced by an operator $T$, namely, $\varrho_{T}(r)=r(T)$, $r \in \operatorname{Rat}\left(\Omega^{*}\right)$, the algebra of rational functions with poles off $\bar{\Omega}^{*}$, we assume that $\|r(T)\| \leq\|r\|_{\Omega^{*}, \infty}$. For such operators $T$, the curvature inequality

$$
\mathscr{K}_{T}(\bar{w}) \leq-4 \pi^{2}\left(S_{\Omega^{*}}(\bar{w}, \bar{w})\right)^{2}, \quad \bar{w} \in \Omega^{*},
$$

where $S_{\Omega^{*}}$ is the Sz̈ego kernel of the domain $\Omega^{*}$, was established in [48]. Equivalently, since $S_{\Omega}(z, w)=$ $S_{\Omega^{*}}(\bar{w}, \bar{z}), z, w \in \Omega$, the curvature inequality takes the form

$$
\frac{\partial^{2}}{\partial w \partial \bar{w}} \log K(w, w) \geq 4 \pi^{2}\left(S_{\Omega}(w, w)\right)^{2}, \quad w \in \Omega .
$$

The curvature inequality in (1.5) is for operators $T$ in $B_{1}\left(\Omega^{*}\right)$ for which $\bar{\Omega}^{*}$ is a spectral set. It is not known if there exists an extremal operator $T$ in $B_{1}\left(\Omega^{*}\right)$, that is, if $\mathscr{K}_{T}(w)=-4 \pi^{2}\left(S_{\Omega^{*}}(w, w)\right)^{2}, w \in \Omega^{*}$, for some operator $T$ in $B_{1}\left(\Omega^{*}\right)$. Indeed, from a result of Suita (cf. [58]), it follows that the adjoint of the multiplication operator on the Hardy space $\left(H^{2}(\Omega), d s\right)$ is not extremal. It was shown in [48] that for any fixed but arbitrary $w_{0} \in \Omega$, there exists an operator $T$ in $B_{1}\left(\Omega^{*}\right)$ for which equality is achieved, at $w=w_{0}$, in the inequality (1.5). The question of Douglas is the question of uniqueness of such an operator. It was partially answered recently in [55]. The precise result is that these "point-wise" extremal operators are determined uniquely within the class of the adjoint of the bundle shifts introduced in [1]. It was also shown in the same paper that each of these bundle shifts can be realized as a multiplication operator on a Hilbert space of weighted Hardy space and conversely. Some very interesting inequalities involving, what the authors call "higher order curvature", are given in [59].

1.3. Homogeneous operators. The question of Douglas discussed before has an affirmative answer in the class of homogeneous operators in the Cowen-Douglas class. An operator $T$ with its spectrum $\sigma(T)$ contained in the closed unit disc $\overline{\mathbb{D}}$ is said to be homogeneous if $U_{\varphi}^{*} T U_{\varphi}=\varphi(T)$ for each bi-holomorphic automorphism $\varphi$ of the unit disc and some unitary $U_{\varphi}$. It is then natural to ask what are all the homogeneous operators. Let us describe (see [47, 60, 7]) the homogeneous operators in $B_{1}(\mathbb{D})$ ). We first show that the equivalence class of a holomorphic Hermitian line bundle $L$ defined on a bounded planar domain $\Omega$ is determined by its curvature $\mathscr{K}_{L}$.

Proposition 1.1. Suppose that $E$ and $F$ are two holomorphic Hermitian line bundles defined on some bounded domain $\Omega \subseteq \mathbb{C}^{m}$. Then they are locally equivalent as holomorphic Hermitian bundles if and only if $\mathscr{K}_{E}=\mathscr{K}_{F}$.

Proof. For simplicity, first consider the case of $m=1$. Suppose that $E$ is a holomorphic line bundle over the domain $\Omega \subseteq \mathbb{C}$ with a hermitian metric $G(w)=\left\langle\gamma_{w}, \gamma_{w}\right\rangle$, where $\gamma$ is a holomorphic frame. The curvature 
$\mathscr{K}_{E}$ is given by the formula $\mathscr{K}_{E}(w)=-\left(\frac{\partial^{2}}{\partial w \partial \bar{w}} \log G\right)(w)$, for $w \in \Omega$. Clearly, in this case, $\mathscr{K}(w) \equiv 0$ on $\Omega$ is the same as saying that $\log G$ is harmonic on $\Omega$. Let $F$ be a second line bundle over the same domain $\Omega$ with the metric $H$ with respect to a holomorphic frame $\eta$. Suppose that the two curvatures $\mathscr{K}_{E}$ and $\mathscr{K}_{F}$ are equal. It then follows that $u=\log (G / H)$ is harmonic on $\Omega$ and thus there exists a harmonic conjugate $v$ of $u$ on any simply connected open subset $\Omega_{0}$ of $\Omega$. For $w \in \Omega_{0}$, define $\tilde{\eta}_{w}=e^{(u(w)+i v(w)) / 2} \eta_{w}$. Then clearly, $\tilde{\eta}_{w}$ is a new holomorphic frame for $F$, which we can use without loss of generality. Consequently, we have the metric $H(w)=\left\langle\tilde{\eta}_{w}, \tilde{\eta}_{w}\right\rangle$ relative to the frame $\tilde{\eta}$ for the vector bundle $F$. We have that

$$
\begin{aligned}
H(w) & =\left\langle\tilde{\eta}_{w}, \tilde{\eta}_{w}\right\rangle \\
& =\left\langle e^{(u(w)+i v(w)) / 2} \eta_{w}, e^{(u(w)+i v(w)) / 2} \eta_{w}\right\rangle \\
& =e^{u(w)}\left\langle\eta_{w}, \eta_{w}\right\rangle \\
& =G(w) .
\end{aligned}
$$

This calculation shows that the map $\tilde{\eta}_{w} \mapsto \gamma_{w}$ defines an isometric holomorphic bundle map between the vector bundles $E$ and $F$.

To complete the proof in the general case, recall that

$$
\sum_{i, j=1}^{m}\left(\frac{\partial^{2}}{\partial w_{i} \partial \bar{w}_{j}} \log G / H\right)(w) d w_{i} \wedge d \bar{w}_{j}=0
$$

means that the function is $u:=H / G$ is pluriharmonic. The proof then follows exactly the same way as in the case of $m=1$. Indeed, as in [18, Theorem 1], the map

$$
U\left(\sum_{|I| \leq n} \alpha_{I}\left(\bar{\partial}^{I} \tilde{\eta}\right)\left(w_{0}\right)\right)=\sum_{|I| \leq n} \alpha_{I}\left(\bar{\partial}^{I} \gamma\right)\left(w_{0}\right), \alpha_{I} \in \mathbb{C},
$$

where $w_{0}$ is a fixed point in $\Omega$ and $I$ is a multi-index of length $n$, is well-defined, extends to a unitary operator on the Hilbert space spanned by the vectors $\left(\bar{\partial}^{I} \tilde{\eta}\right)\left(w_{0}\right)$ and intertwines the two $m$-tuples of operators in $\mathrm{B}_{1}(\Omega)$ corresponding to the vector bundles $E$ and $F$.

As shown in [17], it now follows that the curvature $\mathscr{K}_{T}$ of an operator $T$ in $B_{1}(\Omega)$ determines the unitary equivalence class of $T$ and conversely.

Theorem (Cowen-Douglas) Two operators $T$ and $\tilde{T}$ belonging to the class $B_{1}(\Omega)$ are unitarily equivalent if and only if $\mathscr{K}_{T}=\mathscr{K}_{\tilde{T}}$.

Proof. Let $\gamma_{T}(w)$ be a holomorphic frame for the line bundle $E_{T}$ over $\Omega$ corresponding to an operator $T$ in $\mathrm{B}_{1}(\Omega)$. Thus the real analytic function $G_{T}(w):=\left\langle\gamma_{T}(w), \gamma_{T}(w)\right\rangle$ is the Hermitian metric for the bundle $E_{T}$. Similarly, let $\gamma_{\tilde{T}}$ and $G_{\tilde{T}}$ be the holomorphic frame and the Hermitian metric corresponding to the operator $\tilde{T}$. If $T$ and $\tilde{T}$ are unitarily equivalent, then the eigenvector $\gamma_{\tilde{T}}(w)$ must be a multiple, say $c$ depending on $w$, of the eigenvector $\gamma_{T}(w)$. However, since both $\gamma_{\tilde{T}}$ and $\gamma_{T}$ are holomorphic, it follows that $c$ must be holomorphic. Hence $G_{\tilde{T}}(w)=|c(w)|^{2} G_{T}(w)$ and we see that $\mathscr{K}_{T}=\mathscr{K}_{\tilde{T}}$.

Conversely, if the two curvatures are equal, from Proposition 1.1, we find that we may choose, without loss of generality, a holomorphic frame $\gamma_{\tilde{T}}$ for the operator $\tilde{T}$ such that $G_{\tilde{T}}=G_{T}$. Since the linear span of the vectors $\gamma_{T}(w)$ and $\gamma_{\tilde{T}}(w)$ are dense, it follows that the map $U$ taking $\gamma_{T}(w)$ to $\gamma_{\tilde{T}}(w)$ is isometric. Extending it linearly, we obtain a unitary operator that intertwines $T$ and $\tilde{T}$

We now explain how the curvature can be extracted directly from an operator $T: \mathscr{H} \rightarrow \mathscr{H}$ which is in the class $B_{1}(\Omega)$. Let $\gamma$ be a holomorphic frame for the operator $T$. Recall that $\gamma^{\prime}(w), w \in \Omega$, is also in the Hilbert space $\mathscr{H}$. The restriction $N(w)$ of the operator $T-w I$ to the two dimensional subspaces $\left\{\gamma(w), \gamma^{\prime}(w)\right\}, w \in \Omega$ is nilpotent and encodes important information about the operator $T$. 
With a little more effort, one may work with commuting tuples of bounded operators on a Hilbert space possessing an open set $\Omega \subseteq \mathbb{C}^{m}$ of joint eigenvalues. We postpone the details to Section 2.1.1.

Let $\mathscr{N}(w) \subseteq \mathscr{H}, w \in \Omega$, be the subspace consisting of the two linearly independent vectors $\gamma(w)$ and $\gamma^{\prime}(w)$. There is a natural nilpotent action $N(w):=(T-w I)_{\mid \mathscr{N}(w)}$ on the space $\mathscr{N}(w)$ determined by the rule

$$
\gamma^{\prime}(w) \stackrel{N(w)}{\longrightarrow} \gamma(w) \stackrel{N(w)}{\longrightarrow} 0 .
$$

Let $e_{0}(w), e_{1}(w)$ be the orthonormal basis for $\mathscr{N}(w)$ obtained from $\gamma(w), \gamma^{\prime}(w)$ by the Gram-Schmidt orthonormalization. The matrix representation of $N(w)$ with respect to this orthonormal basis is of the form $\left(\begin{array}{cc}0 & h(w) \\ 0 & 0\end{array}\right)$. It is easy to compute $h(w)$. Indeed, we have

$$
h(w)=\frac{\|\gamma(w)\|^{2}}{\left(\left\|\gamma^{\prime}(w)\right\|^{2}\|\gamma(w)\|^{2}-\left|\left\langle\gamma^{\prime}(w), \gamma(w)\right\rangle\right|^{2}\right)^{\frac{1}{2}}} .
$$

We observe that $\mathscr{K}_{T}(w)=-h(w)^{-2}$.

Let $\varphi$ be a bi-holomorphic automorphism of the unit disc $\mathbb{D}$. Thus $\varphi(z)$ is of the form $e^{i \theta} \frac{z-\alpha}{1-\bar{\alpha} z}$ for some $\theta, \alpha, 0 \leq \theta<2 \pi, \alpha \in \mathbb{D}$. Since the spectrum of $T$ is contained in $\overline{\mathbb{D}}$ and $q(z)=1-\bar{\alpha} z$ does not vanish on it, we can define $\varphi(T)$ to be the operator $p(T) q(T)^{-1}$, where $p(z)=z-\alpha$. This definition coincides with the usual holomorphic functional calculus. It is not hard to prove that $\varphi(T)$ is in $B_{1}(\mathbb{D})$, whenever $T$ is in $B_{1}(\mathbb{D})$, see [50].

Theorem 1.1. Let $T$ be an operator in $\mathrm{B}_{1}(\mathbb{D})$. Suppose that $\varphi(T)$ unitarily equivalent to $T$ for each biholomorphic automorphism of $\varphi$ of $\mathbb{D}$. Then

$$
\mathscr{K}_{T}(\alpha)=\left(1-|\alpha|^{2}\right)^{-2} \mathscr{K}_{T}(0),
$$

where $-\lambda=\mathscr{K}_{T}(0)<0$ is arbitrary.

Proof. For each fixed but arbitrary $w \in \mathbb{D}$, we have

$$
\varphi(T)_{\mid \operatorname{ker}(\varphi(T)-\varphi(w))^{2}}=\varphi\left(T_{\mid \operatorname{ker}(T-w)^{2}}\right) .
$$

Since $T_{\mid \operatorname{ker}(T-w)^{2}}$ is of the form $\left(\begin{array}{cc}w & h_{T}(w) \\ 0 & w\end{array}\right)$ and

$$
\varphi\left(\begin{array}{cc}
w & h_{T}(w) \\
0 & w
\end{array}\right)=\left(\begin{array}{cc}
\varphi(w) & \varphi^{\prime}(w) h_{T}(w) \\
0 & \varphi(w)
\end{array}\right)
$$

it follows that

$$
\varphi(T)_{\mid \operatorname{ker}(\varphi(T)-\varphi(w))^{2}}=\left(\begin{array}{cc}
\varphi(w) & \varphi^{\prime}(w) h_{T}(w) \\
0 & \varphi(w)
\end{array}\right) \cong\left(\begin{array}{cc}
\varphi(w) & \left|\varphi^{\prime}(w)\right| h_{T}(w) \\
0 & \varphi(w)
\end{array}\right)
$$

where we have used the symbol $\cong$ for unitary equivalence. Finally, we have

$$
\begin{aligned}
\left(-\mathscr{K}_{\varphi(T)}(\varphi(w))\right)^{-1 / 2} & =h_{\varphi(T)}(\varphi(w)) \\
& =\left|\varphi^{\prime}(w)\right| h_{T}(w) \\
& =\left|\varphi^{\prime}(w)\right|\left(-\mathscr{K}_{T}(w)\right)^{-1 / 2} .
\end{aligned}
$$

This is really a "change of variable formula for the curvature", which can be obtained directly using the chain rule.

Put $w=0$, choose $\varphi=\varphi_{\alpha}$ such that $\varphi(0)=\alpha$. In particualr, take $\varphi_{\alpha}(z)=\frac{\alpha-z}{1-\bar{\alpha} z}$. Then

$$
\begin{aligned}
& \mathscr{K}_{\varphi_{\alpha}(T)}(\alpha)=\mathscr{K}_{\varphi_{\alpha}(T)}\left(\varphi_{\alpha}(0)\right)=\left|\varphi^{\prime}(0)\right|^{-2} \mathscr{K}_{T}(0) \\
&=\left(1-|\alpha|^{2}\right)^{-2} \mathscr{K}_{T}(0), \quad \alpha \in \mathbb{D} . \\
& 8
\end{aligned}
$$


Now, suppose that $\varphi_{\alpha}(T)$ is unitarily equivalent to $T$ for all $\varphi_{\alpha}, \alpha \in \mathbb{D}$. Then $K_{\varphi_{\alpha}(T)}(w)=\mathscr{K}_{T}(w)$ for all $w \in \mathbb{D}$. Hence

$$
\left(1-|\alpha|^{2}\right)^{-2} \mathscr{K}_{T}(0)=K_{\varphi_{\alpha}(T)}\left(\varphi_{\alpha}(0)\right)=K_{T}(\alpha), \quad \alpha \in \mathbb{D} .
$$

(Here the first equality is the change of variable formula given in (1.7) and the second equality follows from equality of the curvature of two unitarily equivalent operators.)

Corollary 1.1. If $T$ is a homogeneous operator in $B_{1}(\mathbb{D})$, then it must be the adjoint of the multiplication operator on the reproducing kernel Hilbert space $\mathscr{H}^{(\lambda)}$ determined by the reproducing kernel $K^{(\lambda)}(z, w):=$ $(1-z \bar{w})^{-2 \lambda}$.

Proof. It follows from the Theorem that if the operator $T$ is homogeneous, then the corresponding metric $G$ for the bundle $E_{T}$, which is determined up to the square of the absolute value of a holomorphic function, is of the form:

$$
G(w)=\left(1-|w|^{2}\right)^{-2 \lambda}, \quad w \in \mathbb{D}, \lambda>0 .
$$

This corresponds to the reproducing kernel $K$ (obtained via polarization of the real analytic function $G$ ) of the form:

$$
K^{(\lambda)}(z, w)=(1-z \bar{w})^{-2 \lambda}, \quad \lambda>0 ; z, w \in \mathbb{D} .
$$

The kernel $B(z, w)=(1-z \bar{w})^{-2}$ is the reproducing kernel of the Hilbert space of square integrable (with respect to area measure) holomorphic functions defined on the unit disc $\mathbb{D}$ and is known as the Bergman kernel. The kernel $K^{(\lambda)}$ is therefore a power of the Bergman kernel and the Hilbert space $\mathscr{P}^{(\lambda)}$ determined by $K^{(\lambda)}$ is known as the weighted Bergman space. The adjoint of the multiplication operator $M$ on the Hilbert space $\mathscr{H}^{(\lambda)}$ corresponding to the reproducing kernel $K^{(\lambda)}$ is in $B_{1}(\mathbb{D})$.

If $\lambda>\frac{1}{2}$, then the multiplication operator $M^{(\lambda)}$ is subnormal and the inner product in the Hilbert space $\mathscr{H}^{(\lambda)}$ is induced by the measure

$$
d \mu(z)=\frac{2 \lambda-1}{\pi}\left(1-|z|^{2}\right)^{2 \lambda-2} d z d \bar{z} .
$$

For $\lambda=\frac{1}{2}$, this operator is the usual shift on the Hardy space. However, for $\lambda<\frac{1}{2}$, there is no such measure and the corresponding operator $M^{(\lambda)}$ is not a contraction, not even power bounded and therefore not subnormal.

1.4. Quotient and submodules. The interaction of one-variable function theory and functional analysis with operator theory over the past half century has been extremely fruitful. Much of the progress in multivariable spectral theory during the last two decades was made possible by the use of methods from several complex variables, complex analytic and algebraic geometry. A unifying approach to many of these problems is possible in the language of Hilbert modules.

For any ring $R$ and an ideal $I \subseteq R$, the study of the pair $I$ and $R / I$ as modules over the ring $R$ is natural in algebra. However, if one were to assume that the ring $R$ has more structure, for instance, if $R$ is taken to be the polynomial ring $\mathbb{C}[z]$, in $m$ - variables, equipped with the supremum norm over some bounded domain $\Omega$ in $\mathbb{C}^{m}$, then the study of a pair analogous to $I$ and $R / I$, as above, still makes sense and is important. Fix an inner product on the algebra $\mathbb{C}[z]$. The completion of $\mathbb{C}[z]$ with respect to this inner product is a Hilbert space, say $\mathscr{M}$. It is natural to assume that the natural action of point-wise multiplication module action $\mathbb{C}[z] \times \mathbb{C}[z] \rightarrow \mathbb{C}[z]$ extends continuously to $\mathbb{C}[z] \times \mathscr{M} \rightarrow \mathscr{M}$ making $\mathscr{M}$ a module over $\mathbb{C}[z]$. Natural examples are the Hardy and Bergman spaces on some bounded domain $\Omega \subseteq \mathbb{C}^{m}$. Here the module map is induced by point-wise multiplication, namely, $\mathbf{m}_{p}(f)=p f, p \in \mathbb{C}[\boldsymbol{z}], f \in \mathscr{M}$. A Hilbert module need not be obtained as the completion of the polynomial ring, more generally, a Hilbert module is simply a Hilbert 
space equipped with an action of a ring $R$. When this action is continuous in both the variables, the Hilbert space $\mathscr{M}$ is said to be a Hilbert module over the polynomial ring $\mathbb{C}[z]$.

A closed subspace $\mathscr{S}$ of $\mathscr{M}$ is said to be a submodule of $\mathscr{M}$ if $\mathbf{m}_{p} h \in \mathscr{S}$ for all $h \in \mathscr{S}$ and $p \in \mathbb{C}[z]$. The quotient module $\mathscr{Q}:=\mathscr{M} / \mathscr{S}$ is the Hilbert space $\mathscr{S}^{\perp}$, where the module multiplication is defined to be the compression of the module multiplication on $\mathscr{M}$ to the subspace $\mathscr{S}^{\perp}$, that is, the module action on $\mathscr{Q}$ is given by $\mathbf{m}_{p}(h)=P_{\mathscr{S}_{\perp}}\left(\mathbf{m}_{p} h\right), h \in \mathscr{S}^{\perp}$. Two Hilbert modules $\mathscr{M}_{1}$ and $\mathscr{M}_{2}$ over $\mathbb{C}[z]$ are said to be isomorphic if there exists a unitary operator $U: \mathscr{M}_{1} \rightarrow \mathscr{M}_{2}$ such that $U(p \cdot h)=p \cdot U h, p \in \mathbb{C}[z], h \in \mathscr{M}_{1}$. A Hilbert module $\mathscr{M}$ over the polynomial ring $\mathbb{C}[\mathbf{z}]$ is said to be in $B_{n}(\Omega)$ if $\operatorname{dim} \mathscr{H} / \mathfrak{m}_{w} \mathscr{M}=n<\infty$ for all $w \in \Omega$, and $\cap_{w \in \Omega} \mathfrak{m}_{w} \mathscr{M}=\{0\}$, where $\mathfrak{m}_{w}$ is the maximal ideal in $\mathbb{C}[\mathbf{z}]$ at $w$. In practice, it is enough to work with analytic Hilbert modules defined below.

Definition 1.2. A Hilbert module $\mathscr{M}$ over $\mathbb{C}[z]$ is called an analytic Hilbert module if the Hilbert space $\mathscr{M}$ consists of holomorphic functions on some bounded domain $\Omega \subseteq \mathbb{C}^{m}, \mathbb{C}[z] \subseteq \mathscr{M}$ is dense in $\mathscr{M}$, and $\mathscr{H}$ possesses a reproducing kernel $K: \Omega \times \Omega \rightarrow \mathscr{L}(V)$, where $V$ is some finite dimensional linear space.

The module action in an analytic Hilbert module $\mathcal{M}$ is given by point-wise multiplication, that is, $\mathfrak{m}_{p}(h)(\boldsymbol{z})=p(\boldsymbol{z}) h(\boldsymbol{z}), \boldsymbol{z} \in \Omega$.

There are many closely related notions like the locally free module and the quasi-free module (cf. [13, 26]). No matter which notion one adopts, the goal is to ensure the existence of a holomorphic Hermitian vector bundle such that the equivalence class of the module and that of the vector bundle are in one to one correspondence. The generalized Bergman kernel and the sharp kernel appearing in [21] and [2] achieve a similar objective. The polynomial density in an analytic Hilbert module ensures that the joint kernel

$$
\cap_{i=1}^{m} \operatorname{ker}\left(M_{i}-w_{i}\right)^{*}, w \in \Omega^{*},
$$

is of constant dimension, see [25, Remark, pp. 5]. Let $K$ be the reproducing kernel of the analytic Hilbert module $\mathscr{M}$ and $\left\{\varepsilon_{i}\right\}_{i=1}^{n}$, be a basis of the linear space $V$. Evidently, the map

$$
\gamma_{i}: w \rightarrow K(\cdot, \bar{w}) \varepsilon_{i}, w \in \Omega^{*}, i=1, \ldots, n,
$$

serves as a holomorphic frame for the Hilbert module $\mathcal{M}$. This way, we obtain a holomorphic Hermitian vector bundle $E_{\mathscr{M}}$.

Let $\mathcal{Z} \subseteq \Omega$ be an analytic submanifold, $T \Omega=T \mathcal{Z}+N \mathcal{Z}$ be the decomposition of the tangent bundle of $\Omega$. Pick a basis for the normal bundle $N \mathscr{Z}$, say $\partial_{i}, 1 \leq i \leq p$. Fix the submodule $\mathscr{M}_{0} \subseteq \mathscr{M}$ of all functions $f$ in $\mathscr{M}$ vanishing on $\mathcal{Z}$ to total order $k$, that is, $\partial^{\boldsymbol{\alpha}} f_{\text {|res } Z}=0$ for all multi index $\boldsymbol{\alpha}$ of length less or equal to $k$. We now have a a short exact sequence

$$
0 \longleftarrow \mathscr{Q} \longleftarrow \mathscr{M} \stackrel{X}{\longleftarrow} \mathscr{M}_{0} \longleftarrow 0,
$$

where $\mathscr{Q}=\mathscr{M} \ominus \mathscr{M}_{0}$ is the quotient module and $X$ is the inclusion map.

One of the fundamental problems is to find a canonical model and obtain a (complete) set of unitary invariants for the quotient module $\mathscr{Q}$.

If the submodule is taken to be the maximal set of functions vanishing on an analytic hypersurface $\mathcal{Z}$ in $\Omega$, then appealing to an earlier result of Aronszajn [4] the following theorem was proved in [24] to analyze the quotient module $\mathscr{Q}$. Set

$$
\mathscr{M}_{\text {res }}=\left\{f_{\mid Z} \mid f \in \mathscr{M}\right\}
$$

and

$$
\left\|f_{\mid Z}\right\|_{\text {res }}=\inf \left\{\|g\|_{\mathscr{M}} \mid g \in \mathscr{M}, g_{\mid Z}=f_{\mid Z}\right\} .
$$


Theorem (Aronszajn). The restriction $\mathscr{M}_{\text {res }}$ is a Hilbert module over $\mathbb{C}[z]$ possessing a reproducing kernel $K_{\text {res }}$, which is the restriction of $K$ to $\mathcal{Z}$, that is, $K_{\text {res }}(\cdot, \boldsymbol{w})=\left.K(\cdot, \boldsymbol{w})\right|_{\mathcal{Z}}$ for $\boldsymbol{w}$ in $\mathfrak{Z}$. Furthermore, $\mathscr{Q}$ is isometrically isomorphic to $\mathscr{M}_{\text {res }}$.

As an example, consider the Hardy module $H^{2}\left(\mathbb{D}^{2}\right)$. Since the Sz̈ego kernel

$$
\mathbb{S}_{\mathbb{D}^{2}}(\mathbf{z}, \mathbf{w})=\frac{1}{\left(1-z_{1} \bar{w}_{1}\right)} \frac{1}{\left(1-z_{2} \bar{w}_{2}\right)}
$$

is the reproducing kernel of $H^{2}\left(\mathbb{D}^{2}\right)$, restricting it to the hyper-surface $z_{1}-z_{2}=0$ and using new coordinates $u_{1}=\frac{z_{1}-z_{2}}{2}, u_{2}=\frac{z_{1}+z_{2}}{2}$, we see that $K_{\mathscr{Q}}(u, v)=\frac{1}{(1-u \bar{v})^{2}}$ for $u, v$ in $\left\{z_{1}-z_{2}=0\right\}$. This is a multiple of the kernel function for the Bergman space $L_{\text {hol }}^{2}(\mathbb{D})$. Hence the quotient module is isometrically isomorphism to the Bergman module since multiplication by a constant doesn't change the isomorphism class of a Hilbert module.

Thus the extension of Aronszajn's result provides a model for the quotient module. However, Hilbert modules determined by different kernel functions may be equivalent. To obtain invariants one approach is to appeal to the inherent complex geometry. Assume that the $m$ - tuple of multiplication operators by the coordinate functions on the Hilbert module $\mathscr{M}$ belongs to $B_{1}(\Omega)$. Then the results from [17] apply and show that the curvature is a complete unitary invariant. Therefore, if the quotient module belongs to $B_{1}(Z)$, we need to compute its curvature. It is shown in [24] that the curvature of $\mathscr{Q}$ is the restriction of the curvature $(1,1)$ form of $\mathcal{M}$ to the hyper-surface $\mathcal{Z}$ followed by a projection to the $(1,1)$ forms on $\mathcal{Z}$.

The submodule in [28] is taken to be the (maximal) set of functions which vanish to some given order $k$ on the hypersurface $\mathcal{Z}$. As in the previous case, two descriptions are provided for the quotient module. The first one, produces a Hilbert space of holomorphic functions taking values in $\mathbb{C}^{k}$ via what is known as the jet construction. The kernel function now takes values in $k \times k$ matrices. The second one provides a rank $k$ holomorphic Hermitian vector bundle. Although, describing a complete set of invaraints is much more complicated in this case (cf. [27]).

In the paper [19], it was observed that all the submodules of the Hardy module are isomorphic to the Hardy module, that is, there exists an intertwining unitary module map between them. Applying the von Neumann-Wold decomposition, it is not difficult to obtain description of all the submodules of the Hardy module. Following this new proof of the Beurling's theorem, it was natural determine isomorphism classes of submodules of other Hilbert modules. For instance, the situation is much more complicated for the Hardy module in $m$ - variables. Indeed, the submodule

$$
H_{0}^{2}\left(\mathbb{D}^{2}\right):=\left\{f \in H^{2}\left(\mathbb{D}^{2}\right) \mid f(0,0)=0\right\}
$$

is not equivalent to the Hardy module. This is easy to see: dimension of the joint kernel $\cap_{i=1,2} \operatorname{ker} M_{i}^{*}$ of the two multiplication operators on $H^{2}\left(\mathbb{D}^{2}\right)$ is 1 , while it is 2 on $H_{0}^{2}\left(\mathbb{D}^{2}\right)$.

The fundamental question of which submodules of a Hilbert module are equivalent was answered in [30] after making reasonable hypothesis on the nature of the submodule. A different approach to the same problem is outlined in [10]. A sheaf model to study submodules like the Hardy module $H_{0}^{2}\left(\mathbb{D}^{2}\right)$ of functions vanishing at $(0,0)$ is given in [11].

1.5. Flag structure. Fix a bounded planar domain $\Omega$. Let $E$ be a holomorphic Hermitian vector bundle of rank $n$ in $\Omega \times \mathscr{H}$. By a well-known theorem of Grauert, every holomorphic vector bundle over a plane domain is trivial. So, there exists a holomorphic frame $\gamma_{1}, \ldots, \gamma_{n}: \Omega \rightarrow \mathscr{H}$. A Hermitian metric on $E$ relative to this frame is given by the formula $G_{\gamma}(w)=\left(\left\langle\gamma_{i}(w), \gamma_{j}(w)\right\rangle\right)$. The curvature of the vector bundle $E$ is a 
complex $(1,1)$ form which is given by the formula

$$
\mathscr{K}_{E}(w)=\sum_{i, j=1}^{m} \mathscr{K}_{i, j}(w) d w_{i} \wedge d \bar{w}_{j}
$$

where

$$
\mathscr{K}_{i, j}(w)=-\frac{\partial}{\partial \bar{w}_{j}}\left(G_{\gamma}^{-1}(w)\left(\frac{\partial}{\partial w_{i}} G_{\gamma}(w)\right)\right) .
$$

It clearly depends on the choice of the frame $\gamma$ except when $n=1$. A complete set of invariants is given in [17, 18]. However, these invariants are not easy to compute. So, finding a tractable set of invariants for a smaller class of vector bundles which is complete would be worthwhile. For instance, in the paper [38], irreducible holomorphic Hermitian vector bundles, possessing a flag structure have been isolated. For these, the curvature together with the second fundamental form (relative to the flag) is a complete set of invariants. As an application, at least for $n=2$, it is shown that the homogeneous holomorphic Hermitian vector bundles are in this class. A complete description of these is then given. This is very similar to the case of $n=1$ except that now the second fundamental form associated with the flag has to be also considered along with the curvature. All the vector bundles in this class and the operators corresponding to them are irreducible. The flag structure they possess by definition is rigid which aids in the construction of a canonical model and in finding a complete set of unitary invariants. The study of commuting tuples of operators in the Cowen-Douglas class possessing a flag structure is under way.

The definition of the smaller class $\mathscr{F} B_{2}(\Omega)$ of operators in $B_{2}(\Omega)$ below is from [38]. We will discuss the class $\mathscr{F} B_{n}(\Omega), n>1$ separately at the end.

Definition 1.3. We let $\mathscr{F} B_{2}(\Omega)$ denote the set of bounded linear operators $T$ for which we can find operators $T_{0}, T_{1}$ in $\mathscr{B}_{1}(\Omega)$ and a non-zero intertwiner $S$ between $T_{0}$ and $T_{1}$, that is, $T_{0} S=S T_{1}$ so that

$$
T=\left(\begin{array}{cc}
T_{0} & S \\
0 & T_{1}
\end{array}\right) .
$$

An operator $T$ in $B_{2}(\Omega)$ admits a decomposition of the form $\left(\begin{array}{cc}T_{0} & S \\ 0 & T_{1}\end{array}\right)$ for some pair of operators $T_{0}$ and $T_{1}$ in $B_{1}(\Omega)$ (cf. [40, Theorem 1.49]) . Conversely, an operator $T$, which admits a decomposition of this form for some choice of $T_{0}, T_{1}$ in $B_{1}(\Omega)$ can be shown to be in $B_{2}(\Omega)$. In defining the new class $\mathscr{F} B_{2}(\Omega)$, we are merely imposing one additional condition, namely that $T_{0} S=S T_{1}$.

An operator $T$ is in the class $\mathscr{F} B_{2}(\Omega)$ if and only if there exist a frame $\left\{\gamma_{0}, \gamma_{1}\right\}$ of the vector bundle $E_{T}$ such that $\gamma_{0}(w)$ and $t_{1}(w):=\frac{\partial}{\partial w} \gamma_{0}(w)-\gamma_{1}(w)$ are orthogonal for all $w$ in $\Omega$. This is also equivalent to the existence of a frame $\left\{\gamma_{0}, \gamma_{1}\right\}$ of the vector bundle $E_{T}$ such that

$$
\frac{\partial}{\partial w}\left\|\gamma_{0}(w)\right\|^{2}=\left\langle\gamma_{1}(w), \gamma_{0}(w)\right\rangle, \quad w \in \Omega .
$$

Our first main theorem on unitary classification is given below.

Theorem 1.2. Let

$$
T=\left(\begin{array}{cc}
T_{0} & S \\
0 & T_{1}
\end{array}\right), \quad \tilde{T}=\left(\begin{array}{cc}
\tilde{T}_{0} & \tilde{S} \\
0 & \tilde{T}_{1}
\end{array}\right)
$$

be two operators in $\mathscr{F} B_{2}(\Omega)$. Also let $t_{1}$ and $\tilde{t}_{1}$ be non-zero sections of the holomorphic Hermitian vector bundles $E_{T_{1}}$ and $E_{\tilde{T}_{1}}$ respectively. The operators $T$ and $\tilde{T}$ are equivalent if and only if $\mathscr{K}_{T_{0}}=\mathscr{K}_{\tilde{T}_{0}}$ (or $\mathscr{K}_{T_{1}}=\mathscr{K}_{\tilde{T}_{1}}$ ) and

$$
\frac{\left\|S\left(t_{1}\right)\right\|^{2}}{\left\|t_{1}\right\|^{2}}=\frac{\left\|\tilde{S}\left(\tilde{t}_{1}\right)\right\|^{2}}{\left\|\tilde{t}_{1}\right\|^{2}}
$$


Cowen and Douglas point out in [18] that an operator in $B_{1}(\Omega)$ must be irreducible. However, determining which operators in $B_{n}(\Omega)$ are irreducible is a formidable task. It turns out that the operators in $\mathscr{F} B_{2}(\Omega)$ are always irreducible. Indeed, if we assume $S$ is invertible, then $T$ is strongly irreducible.

Recall that an operator $T$ in the Cowen-Douglas class $B_{n}(\Omega)$, up to unitary equivalence, is the adjoint of the multiplication operator $M$ on a Hilbert space $\not{H}$ consisting of holomorphic functions on $\Omega^{*}:=\{\bar{w}:$ $w \in \Omega$ \} possessing a reproducing kernel $K$. What about operators in $\mathscr{F} B_{n}(\Omega)$ ? For $n=2$, a model for these operators is described below.

For an operator $T \in \mathscr{F} B_{2}(\Omega)$, there exists a holomorphic frame $\gamma=\left(\gamma_{0}, \gamma_{1}\right)$ with the property $\gamma_{1}(w):=$ $\frac{\partial}{\partial w} \gamma_{0}(w)-t_{1}(w)$ and that $t_{1}(w)$ is orthogonal to $\gamma_{0}(w), w \in \Omega$, for some holomorphic map $t_{1}: \Omega \rightarrow \not{H}$. In what follows, we fix a holomorphic frame with this property. Then the operator $T$ is unitarily equivalent to the adjoint of the multiplication operator $M$ on a Hilbert space $\mathscr{H}_{\Gamma} \subseteq \operatorname{Hol}\left(\Omega^{*}, \mathbb{C}^{2}\right)$ possessing a reproducing kernel $K_{\Gamma}: \Omega^{*} \times \Omega^{*} \rightarrow \mathscr{M}_{2}(\mathbb{C})$. The details are in [38]. It is easy to write down the kernel $K_{\Gamma}$ explictly: For $z, w \in \Omega^{*}$, we have

$$
\begin{aligned}
K_{\Gamma}(z, w) & =\left(\begin{array}{ll}
\left\langle\gamma_{0}(\bar{w}), \gamma_{0}(\bar{z})\right\rangle & \left\langle\gamma_{1}(\bar{w}), \gamma_{0}(\bar{z})\right\rangle \\
\left\langle\gamma_{0}(\bar{w}), \gamma_{1}(\bar{z})\right\rangle & \left\langle\gamma_{1}(\bar{w}), \gamma_{1}(\bar{z})\right\rangle
\end{array}\right) \\
& =\left(\begin{array}{cc}
\left\langle\gamma_{0}(\bar{w}), \gamma_{0}(\bar{z})\right\rangle & \frac{\partial}{\partial \bar{w}}\left\langle\gamma_{0}(\bar{w}), \gamma_{0}(\bar{z})\right\rangle \\
\frac{\partial}{\partial z}\left\langle\gamma_{0}(\bar{w}), \gamma_{0}(\bar{z})\right\rangle & \frac{\partial^{2}}{\partial z \partial \bar{w}}\left\langle\gamma_{0}(\bar{w}), \gamma_{0}(\bar{z})\right\rangle+\left\langle t_{1}(\bar{w}), t_{1}(\bar{z})\right\rangle
\end{array}\right), w \in \Omega .
\end{aligned}
$$

Setting $K_{0}(z, w)=\left\langle\gamma_{0}(\bar{w}), \gamma_{0}(\bar{z})\right\rangle$ and $K_{1}(z, w)=\left\langle t_{1}(\bar{w}), t_{1}(\bar{z})\right\rangle$, we see that the reproducing kernel $K_{\Gamma}$ has the form:

$$
K_{\Gamma}(z, w)=\left(\begin{array}{cc}
K_{0}(z, w) & \frac{\partial}{\partial \bar{w}} K_{0}(z, w) \\
\frac{\partial}{\partial z} K_{0}(z, w) & \frac{\partial^{2}}{\partial z \partial \bar{w}} K_{0}(z, w)+K_{1}(z, w)
\end{array}\right) .
$$

All the irreducible homogeneous operators in $B_{2}(\mathbb{D})$ belong to the class $\mathscr{F} B_{2}(\mathbb{D})$. An application of Theorem 1.2 determines the curvature and the second fundamental form of these operators. It is then not hard to identify these operators (up to unitary equivalence) as shown in [38].

\section{SOME FUtURE DiRECTIONS AND FURTHER THOUGHTS}

In this second half of the paper, we discuss some of the topics mentioned only briefly in the first half. Along the way, we also mention some of the open problems in these topics.

2.1. Operators in the Cowen-Douglas class. In this section, we give a description of the local operators $\boldsymbol{T}_{\mid \mathscr{N}(w)}$, where

$$
\mathscr{N}(w):=\cap_{i, j=1}^{m} \operatorname{ker}\left(T_{i}-w_{i} I\right)\left(T_{j}-w_{j} I\right) .
$$

The matrix representation of these local operators contains one of the most important geometric invariant, namely, the curvature. Over the past three decades, this has been used to obtain various curvature inequalities. We also discuss a class of pure subnormal operators $T$ studied in detail by Abrahamse an Douglas, see [1]. We describe briefly, a new realization of such operators of rank 1 from the paper [55], as multiplication operators on ordinary weighted Hardy spaces. For operators of rank $>1$, such a description perhaps exists but has not been found yet.

2.1.1. Local operators. Fix a $m$ - tuple of operators $\boldsymbol{T}$ in $\mathrm{B}_{1}(\Omega)$ and let $N_{\boldsymbol{T}}(w)$ be the $m$-tuple of operators $\left(N_{1}(w), \ldots, N_{m}(w)\right)$, where $N_{i}(w)=\left.\left(T_{i}-w_{i} I\right)\right|_{\mathcal{N}(w)}, i=1, \ldots, m$. Clearly, $N_{i}(w) N_{j}(w)=0$ for all $1 \leq$ $i, j \leq m$. Since $\left(T_{i}-w_{i} I\right) \gamma(w)=0$ and $\left(T_{i}-w_{i} I\right)\left(\partial_{j} \gamma\right)(w)=\delta_{i, j} \gamma(w)$ for $1 \leq i, j \leq m$, we have the matrix representation $N_{k}(w)=\left(\begin{array}{cc}0 & e_{k} \\ 0 & 0\end{array}\right)$, where $e_{k}$ is the vector $(0, \ldots, 1, \ldots, 0)$ with the 1 in the $k$ th slot, $k=1, \ldots, m$. 
Representing $N_{k}(w)$ with respect to an orthonormal basis in $\mathscr{N}(w)$, it is possible to read off the curvature of $\boldsymbol{T}$ at $w$ using the relationship:

$$
\left(-\mathscr{K}_{\boldsymbol{T}}(w)^{\mathrm{t}}\right)^{-1}=\left(\operatorname{tr}\left(N_{k}(w){\overline{N_{j}(w)}}^{\mathrm{t}}\right)\right)_{k, j=1}^{m}=A(w)^{\mathrm{t}} \overline{A(w)},
$$

where the $k$ th-column of $A(w)$ is the vector $\boldsymbol{\alpha}_{k}$ (depending on $w$ ) which appears in the matrix representation of $N_{k}(w)$ with respect to an appropriate choice of an orthonormal basis in $\mathscr{N}(w)$ which we describe below.

This formula is established for a pair of operators in $B_{1}(\Omega)$ (cf. [18, Theorem 7]). However, let us verify it for an $m$-tuple $\boldsymbol{T}$ in $\mathrm{B}_{1}(\Omega)$ for any $m \geq 1$ following [49].

Fix $w_{0}$ in $\Omega$. We may assume without loss of generality that $\left\|\gamma\left(w_{0}\right)\right\|=1$. The function $\left\langle\gamma(w), \gamma\left(w_{0}\right)\right\rangle$ is invertible in some neighborhood of $w_{0}$. Then setting $\hat{\gamma}(w):=\left\langle\gamma(w), \gamma\left(w_{0}\right)\right\rangle^{-1} \gamma(w)$, we see that

$$
\left\langle\partial_{k} \hat{\gamma}\left(w_{0}\right), \gamma\left(w_{0}\right)\right\rangle=0, k=1,2, \ldots, m \text {. }
$$

Thus $\hat{\gamma}$ is another holomorphic section of $E$. The norms of the two sections $\gamma$ and $\hat{\gamma}$ differ by the absolute square of a holomorphic function, that is

$$
\frac{\|\hat{\gamma}(w)\|}{\|\gamma(w)\|}=\left|\left\langle\gamma(w), \gamma\left(w_{0}\right)\right\rangle\right| .
$$

Hence the curvature is independent of the choice of the holomorphic section. Therefore, without loss of generality, we will prove the claim assuming, for a fixed but arbitrary $w_{0}$ in $\Omega$, that

(i) $\left\|\gamma\left(w_{0}\right)\right\|=1$,

(ii) $\gamma\left(w_{0}\right)$ is orthogonal to $\left(\partial_{k} \gamma\right)\left(w_{0}\right), k=1,2, \ldots, m$.

Let $G$ be the Grammian corresponding to the $m+1$ dimensional space spanned by the vectors

$$
\left\{\gamma\left(w_{0}\right),\left(\partial_{1} \gamma\right)\left(w_{0}\right), \ldots,\left(\partial_{m} \gamma\right)\left(w_{0}\right)\right\}
$$

This is just the space $\mathscr{N}\left(w_{0}\right)$. Let $v, w$ be any two vectors in $\mathscr{N}\left(w_{0}\right)$. Find $\boldsymbol{c}=\left(c_{0}, \ldots, c_{m}\right), \boldsymbol{d}=\left(d_{0}, \ldots, d_{m}\right)$ in $\mathbb{C}^{m+1}$ such that $v=\sum_{i=0}^{m} c_{i} \partial_{i} \gamma\left(w_{0}\right)$ and $w=\sum_{j=0}^{m} d_{j} \partial_{j} \gamma\left(w_{0}\right)$. Here $\left(\partial_{0} \gamma\right)\left(w_{0}\right)=\gamma\left(w_{0}\right)$. We have

$$
\begin{aligned}
\langle v, w\rangle & =\left\langle\sum_{i=0}^{m} c_{i} \partial_{i} \gamma\left(w_{0}\right), \sum_{j=0}^{m} d_{j} \partial_{j} \gamma\left(w_{0}\right)\right\rangle \\
& =\left\langle G^{\mathrm{t}}\left(w_{0}\right) \boldsymbol{c}, \boldsymbol{d}\right\rangle_{\mathbb{C}^{m+1}} \\
& =\left\langle\left(G^{\mathrm{t}}\right)^{\frac{1}{2}}\left(w_{0}\right) \boldsymbol{c},\left(G^{\mathrm{t}}\right)^{\frac{1}{2}}\left(w_{0}\right) \boldsymbol{d}\right\rangle_{\mathbb{C}^{m+1}} .
\end{aligned}
$$

Let $\left\{\varepsilon_{i}\right\}_{i=0}^{m}$ be the standard orthonormal basis for $\mathbb{C}^{m+1}$. Also, let $\left(G^{\mathrm{t}}\right)^{-\frac{1}{2}}\left(w_{0}\right) \varepsilon_{i}:=\boldsymbol{\alpha}_{i}\left(w_{0}\right)$, where $\boldsymbol{\alpha}_{i}(j)\left(w_{0}\right)=$ $\alpha_{j i}\left(w_{0}\right), i=0,1, \ldots, m$. We see that the vectors $\varepsilon_{i}:=\sum_{j=0}^{m} \alpha_{j i}\left(\partial_{j} \gamma\right)\left(w_{0}\right), i=0,1, \ldots, m$, form an orthonormal basis in $\mathscr{N}\left(w_{0}\right)$ :

$$
\begin{aligned}
\left\langle\varepsilon_{i}, \varepsilon_{l}\right\rangle & =\left\langle\sum_{j=0}^{m} \alpha_{j i} \partial_{j} \gamma\left(w_{0}\right), \sum_{p=0}^{m} \alpha_{p l} \partial_{p} \gamma\left(w_{0}\right)\right\rangle \\
& =\left\langle\left(G^{\mathrm{t}}\right)^{-\frac{1}{2}}\left(w_{0}\right) \boldsymbol{\alpha}_{i},\left(G^{\mathrm{t}}\right)^{-\frac{1}{2}}\left(w_{0}\right) \boldsymbol{\alpha}_{l}\right\rangle \\
& =\delta_{i l},
\end{aligned}
$$


where $\delta_{i l}$ is the Kronecker delta. Since $N_{k}\left(\left(\partial_{j} \gamma\right)\left(w_{0}\right)\right)=\gamma\left(w_{0}\right)$ for $j=k$ and 0 otherwise, we have $N_{k}\left(\varepsilon_{i}\right)=$ $\left(\begin{array}{cc}0 & \boldsymbol{\alpha}_{k}^{\mathrm{t}} \\ 0 & 0\end{array}\right)$. Hence

$$
\begin{aligned}
\operatorname{tr}\left(N_{i}\left(w_{0}\right) N_{j}^{*}\left(w_{0}\right)\right) & =\boldsymbol{\alpha}_{i}\left(w_{0}\right)^{\mathrm{t}} \overline{\boldsymbol{\alpha}}_{j}\left(w_{0}\right) \\
& =\left(\left(G^{\mathrm{t}}\right)^{-\frac{1}{2}}\left(w_{0}\right) \varepsilon_{i}\right)^{\mathrm{t}} \overline{\left(\left(G^{\mathrm{t}}\right)^{-\frac{1}{2}}\left(w_{0}\right) \varepsilon_{j}\right)} \\
& =\left\langle G^{-\frac{1}{2}}\left(w_{0}\right) \varepsilon_{i}, G^{-\frac{1}{2}}\left(w_{0}\right) \varepsilon_{j}\right\rangle=\left(G^{\mathrm{t}}\right)^{-1}\left(w_{0}\right)_{i, j} .
\end{aligned}
$$

Since the curvature, computed with respect to the holomorphic section $\gamma$ satisfying the conditions (i) and (ii), is of the form

$$
\begin{aligned}
-\mathscr{K}_{\boldsymbol{T}}\left(w_{0}\right)_{i, j} & =\frac{\partial^{2}}{\partial w_{i} \partial \bar{w}_{j}} \log \|\gamma(w)\|_{\mid w=w_{0}}^{2} \\
& =\left(\frac{\|\gamma(w)\|^{2}\left(\frac{\partial^{2} \gamma}{\partial w_{i} \partial \bar{w}_{j}}\right)(w)-\left(\frac{\partial \gamma}{\partial w_{i}}\right)(w)\left(\frac{\partial \gamma}{\partial \bar{w}_{j}}\right)(w)}{\|\gamma(w)\|^{4}}\right)_{\mid w=w_{0}} \\
& =\left(\frac{\partial^{2} \gamma}{\partial w_{i} \partial \bar{w}_{j}}\right)\left(w_{0}\right)=G\left(w_{0}\right)_{i, j}
\end{aligned}
$$

we have verified the claim (2.1).

The local description of the $m$ - tuple of operators $\boldsymbol{T}$ shows that the curvature is indeed obtained from the holomorphic frame and the first order derivatives using the Gram-Schmidt orthonormalization. The following theorem was proved for $m=2$ in (cf. [18, Theorem 7]). However, for any natural number $m$, the proof is evident from the preceding discussion. The case of a $m$-tuple of operators in $B_{n}(\Omega)$ for an arbitrary $n \in \mathbb{N}$ is discussed in [50].

Theorem 2.1. Two m-tuples of operators $\boldsymbol{T}$ and $\tilde{\boldsymbol{T}}$ in $\mathrm{B}_{1}(\Omega)$ are unitarily equivalent if and only if $N_{k}(w)$ and $\tilde{N}_{k}(w), 1 \leq k \leq m$, are simultaneously unitarily equivalent for $w$ in some open subset of $\Omega$.

Proof. Let us fix an arbitrary point $w$ in $\Omega$. In what follows, the dependence on this $w$ is implicit. Suppose that there exists a unitary operator $U: \mathscr{N} \rightarrow \widetilde{\mathcal{N}}$ such that $U N_{i}=\tilde{N}_{i} U, i=1, \ldots, m$. For $1 \leq i, j \leq m$, we have

$$
\begin{aligned}
\operatorname{tr}\left(\tilde{N}_{i} \tilde{N}_{j}^{*}\right) & =\operatorname{tr}\left(\left(U N_{i} U^{*}\right)\left(U N_{j} U^{*}\right)^{*}\right) \\
& =\operatorname{tr}\left(U N_{i} N_{j}^{*} U^{*}\right) \\
& =\operatorname{tr}\left(N_{i} N_{j}^{*} U^{*} U\right) \\
& =\operatorname{tr}\left(N_{i} N_{j}^{*}\right) .
\end{aligned}
$$

Thus the curvature of the operators $\boldsymbol{T}$ and $\tilde{\boldsymbol{T}}$ coincide making them unitarily equivalent proving the Theorem in one direction. In the other direction, observe that if the operators $\boldsymbol{T}$ and $\tilde{\boldsymbol{T}}$ are unitarily equivalent then this unitary must map $\mathscr{N}$ to $\tilde{N}$. Thus the restriction of $U$ to the subspace $\mathscr{N}$ intertwines $N_{k}$ and $\tilde{N}_{k}$ simultaneously for $k=1, \cdots, m$.

2.1.2. Pure subnormal operators. The unilateral shift $U_{+}$or the multiplication $M$ by the coordinate function on the Hardy space $H^{2}(\mathbb{D})$ is a very special kind of subnormal operator in that it is a pure isometry. The spectrum of its minimal normal extension, namely, the bi-lateral shift $U$ or the operator of multiplication $M$ by the coordinate function on $L^{2}(\mathbb{T})$ is the unit circle $\mathbb{T}=\partial \overline{\mathbb{D}}=\partial \sigma\left(U_{+}\right)$. These two properties determine such an operator uniquely, that is, if a pair consisting of a pure isometry $S$ and its minimal normal extension $N$ has the spectral inclusion property: $\sigma(N) \subseteq \partial \sigma(S)$, then $S$ must be unitarily equivalent to the direct sum of a number of copies of the operator $U_{+}$. The situation for the annulus is more complicated and was investigated in [57]. Following this, Abrahamse and Douglas initiated the study of a class of pure subnormal operators $S$ 
which share this property. Thus the spectrum $\sigma(S)$ of the operator $S$ is assumed to be a subset of the closure $\bar{\Omega}$ of a bounded domain $\Omega$ and the spectrum $\sigma(N)$ of its normal extension $N$ is contained in $\partial \sigma(S)$.

Let $\Omega$ be a bounded domain in $\mathbb{C}$ and let $\mathscr{O}(\Omega)$ be the space of holomorphic functions on $\Omega$. For $f \in \mathscr{O}(\Omega)$, the real analytic function $|f|$ is subharmonic and hence admits a least harmonic majorant, which is the function

$$
u_{f}(z):=\inf \{u(z):|f| \leq u, u \text { is harmonic on } \Omega\},
$$

that is, $u_{f}$ is either harmonic or infinity throughout $\Omega$.

Fix a point $w \in \Omega$. The Hardy space on $\Omega$ is defined to be the Hilbert space

$$
H^{2}(\Omega):=\left\{f \in \mathscr{O}(\Omega): u_{f}(w)<\infty\right\} .
$$

It is easily verified that $\|f\|:=u_{f}(w)$ defines a norm and a different choice of $w \in \Omega$ induces an equivalent topology. Let $\mathscr{O}_{\mathfrak{h}}(\Omega)$ denote the space of holomorphic functions $f$ defined on $\Omega$ taking values in some Hilbert space $\mathfrak{h}$. Again, $z \mapsto\|f(z)\|_{\mathfrak{h}}$ is a subharmoinic function and admits a least harmonic majorant $u_{f}$. We define the Hardy space

$$
H_{\mathfrak{h}}^{2}(\Omega):=\left\{f \in \mathscr{O}_{\mathfrak{h}}(\Omega):\|f\|_{w}:=u_{f}(w)<\infty\right\}
$$

as before. Let $m$ be the harmonic measure relative to the point $w \in \Omega$ and let $L^{2}(\partial \Omega, m)$ denote the space of square integrable functions defined on $\partial \Omega$ with respect to $m$. The closed subspace

$$
H^{2}(\partial \Omega):=\left\{f \in L^{2}(\partial \Omega, m): \int_{\partial \Omega} f g d m=0, g \in \mathscr{A}(\Omega)\right\},
$$

where $\mathscr{A}(\Omega)$ is the space of all functions which are holomorphic in some open neighbourhood of the closed set $\bar{\Omega}$, is the Hardy space of $\partial \Omega$. Let $L_{\mathfrak{h}}^{2}(\partial \Omega)$ denote the space of square integrable functions, with respect to the measure $m$, taking values in the Hilbert space $\mathfrak{h}$. The Hardy space $H_{\mathfrak{h}}^{2}(\partial \Omega)$ is the closed subspace of $L_{\mathfrak{h}}^{2}(\partial \Omega)$ consisting of those functions in $L_{\mathfrak{h}}^{2}(\partial \Omega)$ for which $\int_{\partial \Omega} f g d m=0, g \in \mathscr{A}(\Omega)$.

Boundary values. A function $f$ in the Hardy space admits a boundary value $\hat{f}$. This means that the $\lim _{z \rightarrow \lambda} f(z)$ exists (almost everywhere relative to $m$ ) as $z$ approaches $\lambda \in \partial \Omega$ through any non-tangential path in $\Omega$. Define the function $\hat{f}: \partial \Omega \rightarrow \mathbb{C}$ by setting $\hat{f}(\lambda)=\lim _{z \rightarrow \lambda} f(z)$. It then follows that the map $f \mapsto \hat{f}$ is an isometric isomorphism between the two Hardy spaces $H^{2}(\Omega)$ and $H^{2}(\partial \Omega)$, see [56]. This correspondence works for the case of $H_{\mathfrak{h}}^{2}(\Omega)$ and $H_{\mathfrak{h}}^{2}(\partial \Omega)$ as well.

A topological space $E$ is said to be a vector bundle of rank $n$ over $\Omega$ if there is a continuous map $p: E \rightarrow \Omega$ such that $E_{z}:=p^{-1}(z)$ is a linear space. A co-ordinate chart relative to an open cover $\left\{U_{s}\right\}$ of the domain $\Omega$ is a set of homeomorphisms $\varphi_{s}: U_{s} \times \mathfrak{h} \rightarrow p^{-1}\left(U_{s}\right)$ such that $\varphi_{s}(z, k)$ is in $E_{z}$ for any fixed $z \in U_{s}$, the restriction $\varphi_{s}(z):=\left.\varphi_{s}\right|_{\{z\} \times \mathfrak{h}}$ is a continuous linear isomorphism. Therefore, $\varphi_{s t}(z):=\varphi_{s}^{-1}(z) \varphi_{t}(z)$ is in $\mathrm{GL}(\mathfrak{h}), z \in U_{s} \cap U_{t}$. If the transition functions $\varphi_{s t}$ are holomorphic for some choice of co-ordinate functions $\varphi_{s}$, then the vector bundle $E$ is said to be a holomorphic vector bundle. A section $f$ of $E$ is said to be holomorphic if $\varphi_{s}^{-1} f$ is a holomorphic function from $U_{s}$ to $\mathfrak{h}$. Finally, if $\varphi_{s}^{-1}(z) \varphi_{t}(z)$ are chosen to be unitary, $z \in U_{s} \cap U_{t}$, then one says that $E$ is a flat unitary vector bundle. Thus for a flat unitary vector bundle $E$, we have that

$$
\left\|\varphi_{s}(z)^{-1} f(z)\right\|_{\mathfrak{h}}=\left\|\varphi_{t}(z)^{-1} f(z)\right\|_{\mathfrak{h}}, \quad z \in U_{s} \cap U_{t} .
$$

This means that the functions

$$
h_{f}^{(s)}(z):=\left\|\varphi_{s}(z)^{-1} f(z)\right\|_{\mathfrak{h}}, \quad z \in U_{s}
$$

agree on the overlaps and therefore define a function $h_{f}^{E}$ on all of $\Omega$. Since $z \mapsto \varphi_{s}(z)^{-1} f(z)$ is holomorphic and subharmonicity is a local property, it follows that $h_{f}$ is subharmonic on $\Omega$. As before, the function $h_{f}^{E}$ 
admits a least harmonic majorant $u_{f}^{E}$ and the Hardy space $H_{E}^{2}(\Omega)$ is the subspace of holomorphic sections $f$ of $E$ for which

$$
\|f\|_{w}^{E}:=u_{f}^{E}(w)<\infty
$$

for some fixed but arbitrary point $w \in \Omega$.

A function $f$ which is bounded and holomorphic on $\Omega$ defines a bounded linear operator $M_{f}$ on the Hardy space $H_{\mathfrak{h}}^{2}(\Omega)$ via pointwise multiplication. Let $T_{\mathfrak{h}}$ denote the multiplication by the coordinate function $z$. It is then evident that $T_{\mathfrak{h}}$ is unitarily equivalent to the tensor product $T \otimes I_{\mathfrak{h}}$, where $T$ is the multiplication induced by the coordinate function $z$ on the Hardy space $H^{2}(\Omega)$. The Hardy space $H_{E}^{2}(\Omega)$ is also invariant under multiplication by any function which is holomorphic and bounded on $\Omega$. In particular, let $T_{E}$ be the operator of multiplication by $z$ on the Hardy space $H_{E}^{2}(\Omega)$. All the three theorems listed below are from [1].

Theorem 2.2. If $\mathfrak{h}$ is a Hilbert space and $E$ is a flat unitary vector bundle over $\Omega$ of rank $\operatorname{dim} \mathfrak{h}$, then $T_{E}$ is similar to $T_{\mathfrak{h}}$, that is there is a bounded invertible operator $L: H_{\mathfrak{h}}^{2}(\Omega) \rightarrow H_{E}^{2}(\Omega)$ with the property $L^{-1} T_{E} L=T_{\mathfrak{h}}$.

Theorem 2.3. The operators $T_{E}$ and $T_{F}$ are unitarily equivalent if and only if $E$ and $F$ are equivalent as flat unitary vector bundles.

Theorem 2.4. If $E$ is a flat unitary vector bundle of dimension $n$, then for $z \in \Omega$, the dimension of the kernel of $\left(T_{E}-z\right)^{*}$ is $n$.

The operator $T_{E}^{*}$ on $H_{E}^{2}(\Omega)$, where $E$ is a flat unitary line bundle is in $B_{1}\left(\Omega^{*}\right)$, see [48, Corollary 2.1]. Consequently, it defines a holomorphic Hermitian line bundle, say $\mathscr{E}$. One of the main questions that remains unanswered is the relationship between the flat unitary bundle $E$ and the holomorphic Hermitian bundle $\mathscr{E}$. Thus we are asking which operators in $B_{1}(\Omega)$ are pure subnormal operator with the spectral inclusion property and conversely, how to find a model for such an operator in $B_{1}(\Omega)$. Any answer involving the intrinsic complex geometry will be very interesting. In spite of the substantial work in [61] and [12], this question remains elusive. We discuss this a little more in Section 2.2.1. Also, the question of commuting tuples of subnormal operators has not been addressed yet. These are very interesting directions for future research.

There is yet another way, which exploits the covering map $\pi: \mathbb{D} \rightarrow \Omega$, to define the Hardy space on $\Omega$. Let $G$ be the group of deck transformations for the covering map $\pi$. Thus $G$ consists of those bi-holomorphic automorphisms $\varphi: \mathbb{D} \rightarrow \mathbb{D}$ satisfying $\pi \circ \varphi=\pi$. A function $f$ on $\mathbb{D}$ is said to be $G$-automorphic if $f \circ \varphi=f$ for $\varphi \in G$. A function $f$ defined on $\mathbb{D}$ is $G$-automorphic if and only if it is of the form $g \circ \pi$ for some function $g$ defined on $\Omega$.

Let $w=\pi(0)$ and $m$ be the harmonic measure on $\partial \Omega$ relative to $w$. This means that $m$ is supported on $\partial \Omega$ and if $u$ is a function which is continuous on $\bar{\Omega}$ and harmonic on $\Omega$, then

$$
u(w)=\int_{\partial \Omega} u d m
$$

For any $f \in L^{1}(\partial \Omega, m)$, it may be shown that

$$
\int_{\partial \Omega} f d m=\int_{\partial \mathbb{D}} f \circ \pi d \mu
$$

where $\mu$ is the Lebesgue measure on $\partial \mathbb{D}$. Thus $m=\mu \circ \pi^{-1}$.

The Hilbert spaces $L_{\mathfrak{h}}^{2}(\partial \Omega), H_{\mathfrak{h}}^{2}(\Omega)$ and $H_{\mathfrak{h}}^{2}(\partial \Omega)$ now lift to closed subspaces of the respective Hilbert spaces on the unit disc $\mathbb{D}$ via the map $f \mapsto f \circ \pi$. 
The dual $\hat{G}$ of the group $G$ is the group of homomorphisms from $G$ into the circle group $\mathbb{T}$. For each character $\alpha \in \hat{G}$, define the Hardy space

$$
H_{\alpha}^{2}(\mathbb{D}):=\left\{f \in H^{2}(\mathbb{D}): f \circ \varphi=\alpha(\varphi) f\right\} .
$$

This is a closed subspace of $H^{2}(\mathbb{D})$ which is invariant under any function which is $G$ - automorphic, holomorphic and bounded on $\mathbb{D}$. The quotient map $\pi$ is such a function and we set $T_{\alpha}$ to be the operator of multiplication by $\pi$ on $H_{\alpha}^{2}(\mathbb{D})$.

We can define vector valued Hardy spaces $H_{\alpha}^{2}(\mathbb{D}, \mathfrak{h})$ in a similar manner except that $\alpha$ is in $\operatorname{Hom}(G, \mathscr{U}(\mathfrak{h}))$, the group of unitary representations of $G$ on the Hilbert space $\mathfrak{h}$. The operator $T_{\alpha}$ on $H_{\alpha}^{2}(\mathbb{D}, \mathfrak{h})$ is multiplication by $\pi$ as before. Since $G$, in this case, is isomorphic to the fundamental group of $\Omega$, there is a bijective correspondence between equivalence classes of the unitary representations of $G$ on $\mathfrak{h}$ and the equivalence classes of flat unitary vector bundles on $\Omega$. Again, the two theorems stated below are from [1].

Theorem 2.5. The operators $T_{\alpha}$ and $T_{\beta}$ are unitarily equivalent if and only if $\alpha$ and $\beta$ are equivalent representations.

Theorem 2.6. If $E$ is the flat unitary vector bundle on $\Omega$ determined by the representation $\alpha$, then the operator $T_{E}$ is unitarily equivalent to the operator $T_{\alpha}$.

The choice of the harmonic measure in the definition of the Hardy space over the domain $\Omega$ may appear to be somewhat arbitrary but it has the advantage of being a conformal invariant and it is closely related to the Greens' function $g$ of $\Omega$ with pole at $t$ :

$$
d m(z)=-\frac{1}{2 \pi} \frac{\partial}{\partial \eta_{z}}(g(z, t)) d s(z), z \in \partial \Omega,
$$

where $g(z, t)$ denotes the Green's function for the domain $\Omega$ at the point $t$ and $\frac{\partial}{\partial \eta_{z}}$ is the directional derivative along the outward normal direction (with respect to the positively oriented $\partial \Omega$ ). Exploiting this, in the paper [50], a description of the bundle shift is given as multiplication operators on Hardy spaces defined on the domain $\Omega$ with respect to a weighted arc length measure. Briefly, starting with a positive continuous function $\lambda$ on $\partial \Omega$, define the weighted measure $\lambda d s$ on $\partial \Omega$. Since the harmonic measure $m$ is boundedly mutually absolutely continuous with respect to the arc length measure $d s$, it follows that $\left(H^{2}(\partial \Omega), \lambda d s\right)$ acquires the structure of a Hilbert space and the operator $M$ on it is a pure, rationally cyclic, subnormal operator with spectrum equal to $\bar{\Omega}$ and the spectrum of the minimal normal extension is $\partial \Omega$. Consequently, the operator $M$ on $\left(H^{2}(\partial \Omega), \lambda d s\right)$ must be unitarily equivalent to the bundle shift $T_{\alpha}$ on $\left(H_{\alpha}^{2}(\Omega), d m\right)$ for some character $\alpha$. In the paper [50], this character has been explicitly described in Equation (2.2). It is then shown that given any character $\alpha$, there exists a function $\lambda$ such that the operator $M$ on $H^{2}(\partial \Omega, \lambda d s)$ is unitarily equivalent to the bundle shift $T_{\alpha}$.

2.2. Curvature inequalities. The local description of the Cowen-Douglas operators $\boldsymbol{T}$, naturally lead to curvature inequalities relate to many well known extremal problems assuming that the homomorphism induced by the operator $\boldsymbol{T}$ is contractive. Among other things, we discuss a) the question of uniqueness of the extremal operators and $b$ ) if the curvature inequalities with additional hypothesis implies contractivity.

2.2.1. The Douglas question. Let

$$
\mathscr{B}[w]:=\left\{f \in \mathscr{O}(\Omega):\|f\|_{\infty}<1, f(w)=0\right\} .
$$

It is well-known that the extremal problem

$$
\sup \left\{\left|f^{\prime}(w)\right|: f \in \mathscr{B}[w]\right\}
$$


admits a solution, say, $F_{w} \in \mathscr{B}[w]$. The function $F_{w}$ is called the Ahlfor's function and maps $\Omega$ onto $\mathbb{D}$ in a $n$ to 1 fashion if the connectivity of the region $\Omega$ is $n$. Indeed, $F_{w}$ is a branched covering map with branch point at $w$. Also, $F_{w}^{\prime}(w)$ is a real analytic function and polarizing it, we get a new function, holomorphic in the first variable and anti-holomorphic in the second, which is the Sz̈ego kernel of the domain $\Omega$. It is also the reproducing kernel of the Hardy space $H^{2}(\Omega, d s)$, which we denote by the symbol $S_{\Omega}$. The relationship of the Hardy space with the solution to the extremal problem (2.2) yields a curvature inequality for contractive homomorphisms $\varrho_{T}$ induced by operators $T$ in $B_{1}(\Omega)$, where $\varrho_{T}(r)=r(T), r \in \operatorname{Rat}(\Omega)$. Recall that the local operator $N(w)+w I=T_{\mid \mathcal{N}(w)}$ is of the form $\left(\begin{array}{cc}w & h(w) \\ 0 & w\end{array}\right)$, where

$$
h(w)=\left(-\mathscr{K}_{T}(w)\right)^{-\frac{1}{2}} .
$$

Consequently,

$$
r\left(T_{\mid \mathscr{N}(w)}\right)=\left(\begin{array}{cc}
r(w) & r^{\prime}(w) h(w) \\
0 & r(w)
\end{array}\right) .
$$

We have the obvious inequalities

$$
\begin{aligned}
& h(w) \sup \left\{\left|r^{\prime}(w)\right| ; r \in \operatorname{Rat}(\Omega), r(w)=0\right\} \\
& =\sup \left\{\left\|\left(\begin{array}{cc}
0 & r^{\prime}(w) h(w) \\
0 & 0
\end{array}\right)\right\|: r \in \operatorname{Rat}(\Omega), r(w)=0\right\} \\
& \leq \sup \left\{\left\|\left(\begin{array}{cc}
r(w) & r^{\prime}(w) h(w) \\
0 & r(w)
\end{array}\right)\right\|: r \in \operatorname{Rat}(\Omega)\right\} \\
& \leq \sup \left\{\left\|\varrho_{T}(r)\right\|: r \in \operatorname{Rat}(\Omega)\right\}
\end{aligned}
$$

The contractivity of $\varrho_{T}$ then gives the curvature inequality

$$
\begin{aligned}
\mathscr{K}_{T}(w) & =-\left(\frac{1}{h(w)}\right)^{2} \\
& \leq-\left(\sup \left\{\left|r^{\prime}(w)\right| ; r \in \operatorname{Rat}(\Omega), r(w)=0\right\}\right)^{2} \\
& \leq-\sup \left\{\left|f^{\prime}(w)\right|: f \in \mathscr{B}[w]\right\} \\
& \leq-S_{\Omega}(w, w)^{2} .
\end{aligned}
$$

If $\Omega$ is the unit disc, then from the Schwarz Lemma, it follows that $F_{w}^{\prime}(w)=\frac{1}{\left(1-|w|^{2}\right)}$. For the unit disc, taking $T=M^{*}$, the adjoint of the multiplication operator on the Hardy space $H^{2}(\mathbb{D})$, we see that we have equality for all $w$ in $\mathbb{D}$ in the string of inequalities $(2.3)$. One can easily see that it makes no difference if we replace the unit disc by any simply connected domain. However, if $\Omega$ is not simply connected, it is shown in [58] that the inequality is strict if we take the operator $T$ to be the adjoint of the multiplication operator on the Hardy space $H^{2}(\Omega, d s)$.

Let $K$ be a positive definite kernel on $\Omega$. Assume that the adjoint of the multiplication operator $M$ is in $B_{1}\left(\Omega^{*}\right)$. What we have shown is that if $\varrho_{M}$ is contractive, then we have the inequality

$$
\mathscr{K}(\bar{w}):=-\frac{\partial^{2}}{\partial w \partial \bar{w}} \log K(w, w) \leq-S_{\Omega}(w, w)^{2}, w \in \Omega,
$$

where $\mathscr{K}$ denotes the curvature of the operator $M^{*}$ on $(\mathscr{H}, K)$. Since the obvious candidate which might have served as an extremal operator, namely, the multiplication operator on the Hardy space $H^{2}(\Omega, d s)$ is not extremal, one may ask if for a fixed but arbitrary $w_{0} \in \Omega$, there exists a kernel $K_{0}$, depending on $w_{0}$, for which $\mathscr{K}\left(w_{0}\right)=-F_{w_{0}}\left(w_{0}\right)^{2}$. The existence of a kernel $K_{0}$ with this property was established in [48], see also [55]. In the paper [55], more is proved. For instance, it is established that the multiplication operator on $\left(\mathscr{H}, K_{0}\right)$ is uniquely determined answering the question of Douglas within the class of pure subnormal operators $T$ with $\sigma(T) \subseteq \bar{\Omega}$ and $\sigma(N) \subseteq \partial \sigma(T)$, where $N$ is the minimal normal extension of $T$. This is Theorem 2.6 of [55]. The question of Douglas has an affirmative answer in a much larger class of operators when considering contractions in $B_{1}(\mathbb{D})$. The main theorem in [50] is reproduced below. 
Theorem 2.7. Fix an arbitrary point $\zeta \in \mathbb{D}$. Let $T$ be an operator in $B_{1}(\mathbb{D})$ such that $T^{*}$ is a 2 hypercontraction. Suppose that the operator $\left(\phi_{\zeta}(T)\right)^{*}$ has the wandering subspace property for an automorphism $\phi_{\zeta}$ of the unit disc $\mathbb{D}$ mapping $\zeta$ to 0 . If $\mathscr{K}_{T}(\zeta)=-\left(1-|\zeta|^{2}\right)^{-2}$, then $T$ must be unitarily equivalent to $U_{+}^{*}$, the backward shift operator.

We believe, the condition in the Theorem requiring the operator $\left(\phi_{\zeta}(T)\right)^{*}$ to have the wandering subspace property must follow from the assumption that $T$ is in $B_{1}(\mathbb{D})$. We haven't been able to prove this yet.

Continuing our previous question of which holomorphic vector bundles come from flat unitary ones, one might have imagined that the operators in $B_{1}(\Omega)$ which are extremal would do the trick. However, it is shown in [55] that many of the bundle shifts cannot be extremal at any point in the domain. This is discussed at the end of Section 3 of the paper [55]. So, this question remains open.

2.2.2. Infinite divisibility. For a contraction in the Cowen-Douglas class $B_{1}(\mathbb{D})$, we have established that $\mathscr{K}_{T}(w) \leq \mathscr{K}_{S^{*}}(w), w \in \mathbb{D}$, where $S$ is the forward shift operator. Clearly, this is equivalent to saying that the restriction of $T$ to the 2 - dimensional subspace $\mathscr{N}_{T}(w)$ spanned by the two vectors $\gamma_{T}(w), \gamma_{T}^{\prime}(w)$ is contractive. Therefore, it is unreasonable to expect that the curvature inequality for an operator $T$ would force it to be contractive. Examples are given in [9]. A natural question is to ask if the curvature inequality can be strengthened to obtain contractivity. Let $K$ be a positive definite kernel and the adjoint of the multiplication operator $M$ is in $B_{1}(\mathbb{D})$. Clearly,

$$
K^{\ddagger}(z, w):=(1-z \bar{w}) K(z, \bar{w})
$$

need not be nnd (unless $M$ is contractive), however, it is Hermitian symmetric, i.e.

$$
K^{\ddagger}(z, w)=\overline{K^{\ddagger}(w, z)} .
$$

Now, observe that the curvature inequality is equivalent to the inequality

But the function

$$
\frac{\partial^{2}}{\partial w \partial \bar{w}} \log K^{\ddagger}(w, w) \geq 0 \text {. }
$$

$$
\frac{\partial^{2}}{\partial w \partial \bar{w}} \log K^{\ddagger}(w, w)
$$

is real analytic, its polarization is a function of two complex variables and it is Hermitian symmetric in those variables. Thus we ask what if we make the stronger assumption that

$$
\frac{\partial^{2}}{\partial w \partial \bar{w}} \log K^{\ddagger}(w, w)
$$

is nnd. For a Hermitian symmetric function $K$, we write $K \geq 0$ to mean that $K$ is nnd. Similarly, this stronger form of the curvature inequality implies $K^{\ddagger}$ must be infinitely divisible, that is, not only $K^{\ddagger}$ is nnd but all its positive real powers $K^{\ddagger}$ are nnd as well. In particular, it follows that the operator $M$ must be contractive. This is Corollary 4.2 of the paper [9] which is reproduced below. For two Hermitian symmetric functions $K_{1}$ and $K_{2}$, the inequality $K_{1} \leq K_{2}$ means $K_{1}-K_{2}$ is nnd.

Theorem 2.8. Let $K$ be a positive definite kernel on the open unit disc $\mathbb{D}$. Assume that the adjoint $M^{*}$ of the multiplication operator $M$ on the reproducing kernel Hilbert space $\mathscr{H}_{K}$ belongs to $B_{1}(\mathbb{D})$. The function

$$
\frac{\partial^{2}}{\partial w \partial \bar{w}} \log \left(\left(1-|w|^{2}\right) K(w, w)\right)
$$

is positive definite, or equivalently

$$
\mathscr{K}_{M^{*}}(w) \leq \mathscr{K}_{S^{*}}(w), w \in \mathbb{D},
$$

if and only if the multiplication operator $M$ is an infinitely divisible contraction. 
2.2.3. The multi-variable case. We say that a commuting tuple of multiplication operators $\boldsymbol{M}$ is an infinitely divisible row contraction if $(1-\langle z, w\rangle) K(z, w)$ is infinitely divisible, that is,

$$
((1-\langle z, w\rangle) K(z, w))^{t}
$$

is positive definite for all $t>0$.

Recall that $\boldsymbol{R}_{m}^{*}$ is the adjoint of the joint weighted shift operator on the Drury-Arveson space $H_{m}^{2}$. The following theorem is a characterization of infinitely divisible row contractions.

Proposition 2.1. ([9, Corollary 4.3]) Let $K$ be a positive definite kernel on the Euclidean ball $\mathbb{B}_{m}$. Assume that the adjoint $\boldsymbol{M}^{*}$ of the multiplication operator $\boldsymbol{M}$ on the reproducing kernel Hilbert space $\mathscr{H}_{K}$ belongs to $B_{1}\left(\mathbb{B}_{m}\right)$. The function

$$
\left(\left(\frac{\partial^{2}}{\partial w_{i} \partial \bar{w}_{j}} \log ((1-\langle w, w\rangle) K(w, w))\right)\right)_{i, j=1}^{m}, \quad w \in \mathbb{B}_{m},
$$

is positive definite, or equivalently

$$
\mathscr{K}_{\boldsymbol{M}^{*}}(w) \leq \mathscr{K}_{\boldsymbol{R}_{m}^{*}}(w), \quad w \in \mathbb{B}_{m},
$$

if and only if the multiplication operator $\boldsymbol{M}$ is an infinitely divisible row contraction.

In the case of the polydisc, we say a commuting tuple $\boldsymbol{M}$ of multiplication by the co-ordinate functions acting on the Hilbert space $\mathscr{H}_{K}$ is infinitely divisible if

$$
\left(S(z, w)^{-1} K(z, w)\right)^{t},
$$

where

$$
S(z, w):=\prod_{i=1}^{m}\left(1-z_{i} \bar{w}_{i}\right)^{-1}, \quad z, w \in \mathbb{D}^{m},
$$

is positive definite for all $t>0$. Every commuting tuple of contractions $\boldsymbol{M}^{*}$ need not be infinitely divisible. Let $\boldsymbol{S}_{m}$ be the commuting $m$ - tuple of the joint weighted shift defined on the Hardy space $H^{2}\left(\mathbb{D}^{m}\right)$.

Corollary 2.1. ([9, Corollary 4.4]) Let $K$ be a positive definite kernel on the polydisc $\mathbb{D}^{m}$. Assume that the adjoint $\boldsymbol{M}^{*}$ of the multiplication operator $\boldsymbol{M}$ on the reproducing kernel Hilbert space $\mathscr{H}_{K}$ belongs to $B_{1}\left(\mathbb{D}^{m}\right)$. The function

$$
\left(\left(\frac{\partial^{2}}{\partial w_{i} \partial \bar{w}_{j}} \log \left(S(w, w)^{-1} K(w, w)\right)\right)\right)_{i, j=1}^{m}, \quad w \in \mathbb{D}^{m},
$$

is positive definite, or equivalently

$$
\mathscr{K}_{\boldsymbol{M}^{*}}(w) \preceq \mathscr{K}_{\boldsymbol{S}_{m}^{*}}(w), \quad w \in \mathbb{D}^{m},
$$

if and only if the multiplication operator $\boldsymbol{M}$ is an infinitely divisible $m$-tuple of row contractions.

Exploiting the explicit description of the local operators $N_{1}(w), \ldots, N_{m}(w)$, a very general curvature inequality for a commuting tuple of operators $\boldsymbol{T}$ in $B_{n}(\Omega)$ is given in [50, Theorem 2.4]. We reproduce below a simple instance of such inequalities taking $\Omega$ to be the unit ball $\mathbb{B}_{m}$ in $\mathbb{C}^{m}$ and setting $n=1$.

Theorem 2.9. ([49, Theorem 4.2]) Let $\theta_{w}$ be a bi-holomorphic automorphism of $\mathbb{B}_{m}$ such that $\theta_{w}(w)=0$. If $\rho_{\boldsymbol{T}}$ is a contractive homomorphism of $\mathscr{O}\left(\mathbb{B}_{m}\right)$ induced by the localization $N_{\mathbf{T}}(w), \boldsymbol{T} \in \mathrm{B}_{1}(\mathbb{B})$, then

$$
\mathscr{K}_{\boldsymbol{T}}(w) \leq-{\overline{\mathrm{D} \theta_{w}(w)}}^{t} D \theta_{w}(w)=\frac{1}{m+1} \nwarrow_{B}(w), w \in \mathbb{B}_{m},
$$

where $\mathscr{K}_{B}$ is the curvature

$$
\left.-\left(\left(\frac{\partial^{2}}{\partial w_{i} \partial \bar{w}_{j}} \log B(w, w)\right)\right)\right)_{i, j=1}^{m}
$$


and

$$
B(z, w)=\left(\frac{1}{1-\langle z, w\rangle}\right)^{m+1}
$$

is the Bergman kernel of the ball $\mathbb{B}_{m}$.

2.3. Homogeneous operators. Let $\mathscr{D}$ be a bounded symmetric domain. The typical examples are the matrix unit ball $\left(\mathbb{C}^{p \times q}\right)_{1}$ of size $p \times q$, which includes the case of the Euclidean ball, i.e., $q=1$. Let $G:=\operatorname{Aut}(\mathscr{D})$ be the bi-holomorphic automorphism group of $\mathscr{D}$.

For the matrix unit ball, $G:=\mathrm{SU}(p, q)$, which consists of all linear automorphisms leaving the form $\left(\begin{array}{cc}I_{n} & 0 \\ 0 & -I_{m}\end{array}\right)$ on $\mathbb{C}^{p+q}$ invariant. Thus $g \in \mathrm{SU}(p, q)$ is of the form $\left(\begin{array}{ll}a & b \\ c & d\end{array}\right)$. The group $\mathrm{SU}(p, q)$ acts on $\left(\mathbb{C}^{p \times q}\right)_{1}$ via the map

$$
g=\left(\begin{array}{ll}
a & b \\
c & d
\end{array}\right): z \mapsto(a z+b z)(c z+d z)^{-1}, z \in\left(\mathbb{C}^{p \times q}\right)_{1} .
$$

This action is transitive. Indeed $\left(\mathbb{C}^{p \times q}\right)_{1} \cong \mathrm{SU}(p, q) / \mathbf{K}$, where $\mathbf{K}$ is the stabilizer of $\mathbf{0}$ in $\left(\mathbb{C}^{p \times q}\right)_{1}$.

When $\mathscr{D}$ is a bounded symmetric domain of dimension $m$ and $\mathscr{H}$ is any Hilbert space, an $m$-tuple $T=\left(T_{1}, \ldots, T_{m}\right)$ of commuting bounded operators acting on $\mathscr{H}$ is said to be homogeneous (cf. [52, 5]) if their joint Taylor spectrum is contained in $\overline{\mathscr{D}}$ and for every holomorphic automorphism $g$ of $\mathscr{D}$, there exists a unitary operator $U_{g}$ such that

$$
g\left(T_{1}, \ldots, T_{m}\right)=\left(U_{g}^{-1} T_{1} U_{g}, \ldots, U_{g}^{-1} T_{m} U_{g}\right) .
$$

Imprimitivity. More generally, let $G$ be a locally compact second countable (lcsc) topological group and $\mathscr{D}$ be a lcsc $G$-space. Suppose that $U: G \rightarrow \mathscr{U}(\mathscr{H})$ is a unitary representation of the group $G$ on the Hilbert space $\mathscr{H}$ and that $\varrho: \mathbf{C}(\mathscr{D}) \rightarrow \mathscr{L}(\mathscr{H})$ is a $*$ - homomorphism of the $C^{*}$ - algebra of continuous functions $\mathbf{C}(\mathscr{D})$ on the algebra $\mathscr{L}(\mathscr{H})$ of all bounded operators acting on the Hilbert space $\mathscr{H}$. Then the pair $(U, \varrho)$ is said to be a representation of the $G$-space $\mathscr{D}$ if

$$
\varrho(g \cdot f)=U(g)^{*} \varrho(f) U(g), f \in \mathbf{C}(\mathscr{D}), g \in G,
$$

where $(g \cdot f)(w)=f\left(g^{-1} \cdot w\right), w \in \mathscr{D}$. This is the generalization due to Mackey of the imprimitivity relation of Frobenius. These are exactly the homogenous commuting tuples of normal operators.

As before, let $\mathbf{K}$ be the stabilizer group of $\mathbf{0}$ in $G$, thus $G / \mathbf{K} \cong \mathscr{D}$, where the identification is obtained via the map: $g \mathbf{K} \rightarrow g \mathbf{0}$. The action of $G$ on $\mathscr{D}$ is evidently transitive. Given any unitary representation $\sigma$ of $\mathbf{K}$, one may associate a representation $\left(U^{\sigma}, \rho^{\sigma}\right)$ of the $G$-space $\mathscr{D}$. The correspondence

$$
\sigma \rightarrow\left(U^{\sigma}, \rho^{\sigma}\right)
$$

is an equivalence of categories. The representation $U^{\sigma}$ is the representation of $G$ induced by the representation $\sigma$ of the group $\mathbf{K}$. For a semi-simple group $G$, induction from the parabolic subgroups is the key to producing irreducible representations. Along with holomorphic induction, this method gives almost all the irreducible unitary representations of the semi-simple group $G$.

We ask what happens if the algebra of continuous functions is replaced by the polynomial ring and the * - homomorphism $\varrho$ is required to be merely a homomorphism of this ring. These are the commuting tuples of homogeneous operators.

2.3.1. Quasi-invariant kernels. Let $\mathscr{M} \subseteq \operatorname{Hol}(\mathscr{D})$ be a Hilbert space possessing a reproducing kernel, say, $K$. These holomorphic imprimitivities are exactly homogeneous operators. Assume that $\mathscr{M}$ is a Hilbert module over the polynomial ring $\mathbb{C}[\mathbf{z}]$. Let $U: G \rightarrow \mathscr{U}(\mathscr{M})$ be a unitary representation. What are the pairs $(U, \rho)$ that satisfy the imprimitivity relation, namely,

$$
U_{g}^{*} \rho(p) U_{g}=\rho\left(\underset{22}{p} g^{-1}\right), g \in G, p \in \mathbb{C}[\mathbf{z}] .
$$


Suppose that the kernel function $K$ transforms according to the rule

$$
J_{g}(z) K(g(z), g(w)) J_{g}(w)^{*}=K(z, w), g \in G, z, w \in \mathscr{D},
$$

for some holomorphic function $J_{g}: \mathscr{D} \rightarrow \mathbb{C}$. Then the kernel $K$ is said to be quasi-invariant, which is equivalent to saying that the map $U_{g}: f \rightarrow J_{g}\left(f \circ g^{-1}\right), g \in G$, is unitary. If we further assume that the $J_{g}: \mathscr{D} \rightarrow \mathbb{C}$ is a cocycle, then $U$ is a homomorphism. The pair $(U, \rho)$ is a representation of the $G$-space $\mathscr{D}$ and conversely.

Therefore, our question becomes that of a characterization of all the quasi-invariant kernels defined on $\mathscr{D}$, or equivalently, finding all the holomorphic cocycles, which is also equivalent to finding all the holomorphic Hermitian homogeneous vector bundles over $\mathscr{D}$.

Let $K: \Omega \times \Omega \rightarrow \mathscr{M}_{m}(\mathbb{C})$ be a kernel function. We will assume the function $K$ is holomorphic in the first variable and anti-holomorphic in the second. For two functions of the form $K\left(\cdot, w_{i}\right) \zeta_{i}, \zeta_{i}$ in $\mathbb{C}^{m}(i=1,2)$ define their inner product by the reproducing property, that is,

$$
\left\langle K\left(\cdot, w_{1}\right) \zeta_{1}, K\left(\cdot, w_{2}\right) \zeta_{2}\right\rangle=\left\langle K\left(w_{2}, w_{1}\right) \zeta_{1}, \zeta_{2}\right\rangle .
$$

This extends to an inner product on the linear span if and only if $K$ is positive definite in the sense that

$$
\begin{aligned}
\sum_{j, k=1}^{n}\left\langle K\left(z_{j}, z_{k}\right) \zeta_{k}, \zeta_{j}\right\rangle & =\left\langle\sum_{k=1}^{n} K\left(\cdot, z_{k}\right) \zeta_{k}, \sum_{j=1}^{n} K\left(\cdot, z_{j}\right) \zeta_{j}\right\rangle \\
& =\| \sum_{k=1}^{n}\left\langle K\left(\cdot, z_{k}\right) \zeta_{k} \|^{2} \geq 0 .\right.
\end{aligned}
$$

Let $G: \Omega \times \Omega \rightarrow \mathscr{M}_{m}(\mathbb{C})$ be the Grammian $G(z, w)=\left(\left\langle e_{p}(w), e_{q}(z)\right\rangle\right)_{p, q}$ of a set of $m$ antiholomorphic functions $e_{p}: \Omega \rightarrow \mathscr{H}, 1 \leq p \leq m$, taking values in some Hilbert space $\mathscr{H}$. Then

$$
\begin{aligned}
\sum_{j, k=1}^{n}\left\langle G\left(z_{j}, z_{k}\right) \zeta_{k}, \zeta_{j}\right\rangle & =\sum_{j, k=1}^{n}\left(\sum_{p q=1}^{m}\left\langle e_{p}\left(z_{k}\right), e_{q}\left(z_{j}\right)\right\rangle \zeta_{k}(p) \overline{\zeta_{j}(q)}\right) \\
& =\left\|\sum_{j, k} \zeta_{k}(p) e_{p}\left(z_{k}\right)\right\|^{2}>0 .
\end{aligned}
$$

We therefore conclude that $G(z, w)^{\mathrm{tr}}$ defines a positive definite kernel on $\Omega$.

For an anti-holomorphic function $s: \Omega \rightarrow \mathbb{C}^{m}$, let us define the norm, at $w,\|s(w)\|^{2}:=\|K(\cdot, w) s(w)\|^{2}$, where the norm on the right hand side is the norm of the Hilbert space $\mathscr{H}$ defined by the positive definite kernel $K$. Let $\varepsilon_{i}, 1 \leq i \leq m$, be the standard basis vectors in $\mathbb{C}^{m}$. We have

$$
\begin{aligned}
\|K(\cdot, w) s(w)\|^{2} & =\left\|\sum_{i} s_{i}(w) K(\cdot, w) \varepsilon_{i}\right\|^{2} \\
& =\sum_{i, j}\left\langle s_{i}(w) K(\cdot, w) \varepsilon_{i}, s_{j}(w) K(\cdot, w) \varepsilon_{j}\right\rangle \\
& =\sum_{i, j} s_{i}(w) \overline{s_{j}(w)}\left\langle K(\cdot, w) \varepsilon_{i}, K(\cdot, w) \varepsilon_{j}\right\rangle \\
& =\sum_{i, j} K(w, w)_{j, i} s_{i}(w) \overline{s_{j}(w)} \\
& =\overline{(s(w))}^{\operatorname{tr}} K(w, w)^{\operatorname{tr}} s(w)
\end{aligned}
$$

For $w$ in $\Omega$, and $p$ in the set $\{1, \ldots, m\}$, let $e_{p}: \Omega \rightarrow \mathscr{H}$ be the antiholomorphic function:

$$
e_{p}(w):=K_{w}(\cdot) \otimes \frac{\partial}{\partial \bar{w}_{p}} K_{w}(\cdot)-\frac{\partial}{\partial \bar{w}_{p}} K_{w}(\cdot) \otimes K_{w}(\cdot) .
$$


Then

$$
\frac{1}{2}\left\langle e_{p}(w), e_{q}(z)\right\rangle=K(z, w) \frac{\partial^{2}}{\partial z_{q} \partial \bar{w}_{p}} K(z, w)-\frac{\partial}{\partial \bar{w}_{p}} K(z, w) \frac{\partial}{\partial z_{q}} K(z, w) .
$$

The curvature of the metric $K$ is given by the $(1,1)$ - form

$$
\sum \frac{\partial^{2}}{\partial w_{q} \partial \bar{w}_{p}} \log K(w, w) d w_{q} \wedge d \bar{w}_{p} .
$$

Set

$$
\mathscr{K}(z, w):=\left(\frac{\partial^{2}}{\partial z_{q} \partial \bar{w}_{p}} \log K(z, w)\right)_{q p} .
$$

Since $2 K(z, w)^{2} \mathscr{K}(z, w)$ is of the form $G(z, w)^{\operatorname{tr}}$, it follows that $K(z, w)^{2} \mathscr{K}(z, w)$ defines a positive definite kernel on $\Omega$ taking values in $\mathscr{M}_{m}(\mathbb{C})$.

Let $\varphi: \Omega \rightarrow \Omega$ be holomorphic and $g: \Omega \rightarrow \mathbb{C}$ be the function $g(w):=K(w, w), w \in \Omega$. Set $h=g \circ \varphi$. Apply the change of variable formula twice. The first time around, we have

$$
\left(\partial_{i} h\right)(w)=\sum_{\ell}\left(\partial_{\ell} g\right)(\varphi(w))\left(\partial_{i} \varphi_{\ell}\right)(w),
$$

differentiating a second time, we have

$$
\left(\bar{\partial}_{j} \partial_{i} h\right)(w)=\sum_{\ell}\left(\sum_{k} \bar{\partial}_{k}\left(\partial_{\ell} g\right)(\varphi(w)) \overline{\partial_{j} \varphi_{k}(w)}\right)\left(\partial_{i} \varphi_{\ell}\right)(w) .
$$

In terms of matrices, we have

$$
\left(\frac{\partial^{2}}{\partial z_{i} \partial \bar{w}_{j}} \log K(\varphi(z), \varphi(w))\right)_{i, j}=\left(\frac{\partial \varphi_{\ell}}{\partial z_{i}}\right)_{i, \ell}\left(\left(\frac{\partial^{2}}{\partial z_{\ell} \partial \bar{w}_{k}} \log K\right)(\varphi(z), \varphi(w))\right)_{\ell, k}\left(\frac{\partial \bar{\varphi}_{k}}{\partial \bar{z}_{j}}\right)_{k, j} .
$$

Equivalently,

$$
\mathscr{K}_{g \circ \varphi}(w)=D \varphi(w)^{\operatorname{tr}} \mathscr{K}_{g}(\varphi(w)) \overline{D \varphi(w)}
$$

If

$$
\operatorname{det}_{\mathbb{C}} D \varphi(w)(g \circ \varphi)(w) \overline{\operatorname{det}_{\mathbb{C}} D \varphi(w)}=g(w),
$$

then $\mathscr{K}_{g \circ \varphi}(w)$ equals $\mathscr{K}_{g}(w)$. Hence we conclude that $\mathscr{K}$ is invariant under the automorphisms $\varphi$ of $\Omega$ in the sense that

$$
D \varphi(w)^{\operatorname{tr}} \mathscr{K}(\varphi(w)) \overline{D \varphi(w)}=\mathscr{K}(w), w \in \Omega .
$$

Let $Q: \Omega \rightarrow \mathscr{M}_{m}(\mathbb{C})$ be a real analytic function such that $Q(w)$ is positive definite for $w \in \Omega$. Let $\mathscr{H}$ be the Hilbert space of holomorphic functions on $\Omega$ which are square integrable with respect to $Q(w) d V(w)$, that is,

$$
\|f\|^{2}:=\int_{\Omega}\langle Q(w) f(w), f(w)\rangle^{2} d V(w),
$$

where $d V$ is the normalized volume measure on $\mathbb{C}^{m}$. Let $U_{\varphi}: \mathscr{H} \rightarrow \mathscr{H}$ be the operator

$$
\left(U_{\varphi} f\right)(z)=m\left(\varphi^{-1}, z\right)\left(f \circ \varphi^{-1}\right)(z)
$$

for some cocycle $m$. The operator $U_{\varphi}$ is unitary if and only if

$$
\begin{aligned}
\left\|U_{\varphi} f\right\|^{2} & =\int\left\langle Q(w)\left(U_{\varphi} f\right)(w),\left(U_{\varphi} f\right)(w)\right\rangle^{2} d V(w) \\
& =\int\left\langle\overline{m\left(\varphi^{-1}, w\right)} \operatorname{tr} Q(w) m\left(\varphi^{-1}, w\right) f\left(\varphi^{-1}(w)\right), f\left(\varphi^{-1}(w)\right)\right\rangle^{2} d V(w) \\
& =\int\langle Q(w) f(w), f(w)\rangle^{2} d V(w),
\end{aligned}
$$


whenever $Q$ transforms according to the rule

$$
{\overline{m\left(\varphi^{-1}, w\right)}}^{\operatorname{tr}} Q(w) m\left(\varphi^{-1}, w\right)=Q\left(\varphi^{-1}(w)\right)\left|\operatorname{det}_{\mathbb{C}}\left(D \varphi^{-1}\right)(w)\right|^{2} .
$$

Set

$$
m\left(\varphi^{-1}, w\right)=D \varphi^{-1}(w)^{\operatorname{tr}}
$$

and

$$
Q^{(\lambda)}(w):=b(w)^{1-\lambda} \mathscr{K}_{g}(w)^{-1}, \quad \lambda>0,
$$

where $b(w)$ is the restriction of the Bergman kernel to the diagonal subset of $\Omega \times \Omega$. Then $Q^{(\lambda)}$ transforms according to the rule (2.4). If for some $\lambda>0$, the Hilbert space $L_{\text {hol }}^{2}\left(\Omega, Q^{(\lambda)} d V\right)$ determined by the measure is nontrivial, then the corresponding reproducing kernel is of the form $b(w)^{\lambda} \mathscr{K}(w)$.

For the Euclidean ball,

$$
L_{\mathrm{hol}}^{2}\left(\mathbb{B}^{m}, Q^{(\lambda)} d V\right) \neq\{0\}
$$

if and only if $\lambda>1$. This means that the polarization of the real analytic function

$$
\begin{aligned}
(1-\langle w, w\rangle)(1-\langle w, w\rangle)^{-\lambda(n+1)} \mathscr{K}(w) & =(1-\langle w, w\rangle)^{-(\lambda-1)(n+1)} \mathscr{K}(w) \\
& =(1-\langle w, w\rangle)^{-(\lambda+1)(n+1)}\left(\left\langle e_{p}(w), e_{q}(w)\right\rangle\right)_{q p}
\end{aligned}
$$

must also be positive definite. Since $\left(\left\langle e_{p}(w), e_{q}(z)\right\rangle\right)_{q p}$ is positive definite, it follows that $\lambda>-1$ will ensure positivity of the kernel

$$
(1-\langle w, w\rangle)^{-\lambda(n+1)} \mathscr{K}(w)=b(w)^{\lambda+2} \operatorname{Adj}\left(I_{n}-z w^{*}\right) .
$$

2.3.2. Classification. We have already described homogeneous operators in the Cowen-Douglas class $B_{1}(\mathbb{D})$ using the curvature invariant. For operators in $B_{k}(\mathbb{D}), k>1$, the curvature alone does not determine the class of the operator. Examples of irreducible homogeneous operators in $B_{k}(\mathbb{D})$ were given in [42] using an intertwining operator $\Gamma$. For the complete classification, we recall the description of homogeneous vector bundles via holomorphic induction, see [41]. Making this explicit in our context, we were able to construct the intertwining operator $\Gamma$ in general [44]. Some of the details are reproduced below from the announcement [43].

Let $\mathfrak{t} \subseteq \mathfrak{g}^{\mathbb{C}}=\mathfrak{s l}(2, \mathbb{C})$ be the algebra $\mathbb{C} h+\mathbb{C} y$, where

$$
h=\frac{1}{2}\left(\begin{array}{cc}
1 & 0 \\
0 & -1
\end{array}\right), \quad y=\left(\begin{array}{ll}
0 & 0 \\
1 & 0
\end{array}\right) .
$$

Linear representations $(\varrho, V)$ of the algebra $\mathfrak{t} \subseteq \mathfrak{g}^{\mathbb{C}}=\mathfrak{s l}(2, \mathbb{C})$, that is, pairs $\varrho(h), \varrho(y)$ of linear transformations satisfying $[\varrho(h), \varrho(y)]=-\varrho(y)$ provide a para-metrization of the homogeneous holomorphic vector bundles.

In obtaining the classification of the homogeneous operators, it is necessary to work with the universal covering group $\tilde{G}$ of the bi-holomorphic automorphism group $G$ of the unit disc. The $\tilde{G}$ - invariant Hermitian structures on the homogeneous holomorphic vector bundle $E$ (making it into a homogeneous holomorphic Hermitian vector bundle), if they exist, are given by $\varrho(\tilde{\mathbb{K}})$ - invariant inner products on the representation space. Here $\tilde{\mathbb{K}}$ is the stabilizer of 0 in $\tilde{G}$.

An inner product can be $\varrho(\tilde{\mathbb{K}})$ - invariant if and only if $\varrho(h)$ is diagonal with real diagonal elements in an appropriate basis. We are interested only in Hermitizable bundles, that is, those that admit a Hermitian structure. So, we will assume without restricting generality, that the representation space of $\varrho$ is $\mathbb{C}^{n}$ and that $\varrho(h)$ is a real diagonal matrix.

Since $[\varrho(h), \varrho(y)]=-\varrho(y)$, we have $\varrho(y) V_{\lambda} \subseteq V_{\lambda-1}$, where $V_{\lambda}=\left\{\xi \in \mathbb{C}^{n}: \varrho(h) \xi=\lambda \xi\right\}$. Hence $\left(\varrho, \mathbb{C}^{n}\right)$ is a direct sum, orthogonal for every $\varrho(\tilde{K})$ - invariant inner product of "elementary" representations, that is, such 
that

$$
\varrho(h)=\left(\begin{array}{ccc}
-\eta I_{0} & & \\
& \ddots & \\
& & -(\eta+m) I_{m}
\end{array}\right) \text { with } I_{j}=I \text { on } V_{-(\eta+j)}=\mathbb{C}^{d_{j}}
$$

and

$$
Y:=\varrho(y)=\left(\begin{array}{ccccc}
0 & & & & \\
Y_{1} & 0 & & & \\
& Y_{2} & 0 & & \\
& & \ddots & \ddots & \\
& & & Y_{m} & 0
\end{array}\right), \quad Y_{j}: V_{-(\eta+j-1)} \rightarrow V_{-(\eta+j)} .
$$

We denote the corresponding elementary Hermitizable bundle by $E^{(\eta, Y)}$.

The Multiplier and Hermitian structures. As in [44] we will use a natural trivialization of $E^{(\eta, Y)}$. In this, the sections of homogeneous holomorphic vector bundle $E^{(\eta, Y)}$ are holomorphic functions $\mathbb{D}$ taking values in $\mathbb{C}^{n}$. The $\tilde{G}$ action is given by $f \mapsto J_{g^{-1}}^{(\eta, Y)}\left(f \circ g^{-1}\right)$ with multiplier

$$
\left.\left(J_{g}^{(\eta, Y)}(z)\right)\right)_{p, \ell}=\left\{\begin{array}{ll}
\frac{1}{(p-\ell) !}\left(-c_{g}\right)^{p-\ell}\left(g^{\prime}\right)(z)^{\eta+\frac{p+\ell}{2}} Y_{p} \cdots Y_{\ell+1} & \text { if } p \geq \ell \\
0 & \text { if } p<\ell
\end{array},\right.
$$

where $c_{g}$ is the analytic function on $\tilde{G}$ which, for $g$ near $e$, acting on $\mathbb{D}$ by $z \mapsto(a z+b)(c z+d)^{-1}$ agrees with $c$.

Proposition 2.2. We have $E^{(\eta, Y)} \equiv E^{\left(\eta^{\prime}, Y^{\prime}\right)}$ if and only if $\eta=\eta^{\prime}$ and $Y^{\prime}=A Y A^{-1}$ with a block diagonal matrix A.

A Hermitian structure on $E^{(\eta, Y)}$ appears as the assignment of an inner product $\langle\cdot, \cdot\rangle_{z}$ on $\mathbb{C}^{n}$ for $z \in \mathbb{D}$. We can write

$$
\langle\zeta, \xi\rangle_{z}=\langle H(z) \zeta, \xi\rangle \text {, with } H(z)>0 .
$$

Homogeneity as a Hermitian vector bundle is equivalent to

$$
J_{g}(z) H(g \cdot z)^{-1} J_{g}(z)^{*}=H(z)^{-1}, g \in G, z \in \mathbb{D} .
$$

The Hermitian structure is then determined by $H=H(0)$ which is a positive block diagonal matrix. We write $\left(E^{(\eta, Y)}, H\right)$ for the vector bundle $E^{(\eta, Y)}$ equipped with the Hermitian structure $H$. We note that $\left(E^{(\eta, Y)}, H\right) \cong\left(E^{\left(\eta, A Y A^{-1}\right)}, A^{*-1} H A\right)$ for any block diagonal invertible $A$. Therefore every homogeneous holomorphic Hermitian vector bundle is isomorphic with one of the form $\left(E^{(\eta, Y)}, I\right)$.

If $E^{(\eta, Y)}$ has a reproducing kernel $K$ which is the case for bundles corresponding to an operator in the Cowen-Douglas class, then $K$ satisfies

$$
K(z, w)=J_{g}(z) K(g z, g w) J_{g}(w)^{*}
$$

and induces a Hermitian structure $H$ given by $H(0)=K(0,0)^{-1}$. Construction of the bundles with reproducing kernel. For $\lambda>0$, let $\mathbb{A}^{(\lambda)}$ be the Hilbert space of holomorphic functions on the unit disc with reproducing kernel $(1-z \bar{w})^{-2 \lambda}$. It is homogeneous under the multiplier $g^{\lambda}(z)$ for the action of $\tilde{G}$. This gives a unitary representation of $\tilde{G}$. Let

$$
\mathbf{A}^{(\eta)}=\oplus_{j=0}^{m} \mathbb{A}^{(\eta+j)} \otimes \mathbb{C}^{d_{j}}
$$

For $f$ in $\mathbf{A}^{(\eta)}$, we denote by $f_{j}$, the part of $f$ in $\mathbb{A}^{(\eta+j)} \otimes \mathbb{C}^{d_{j}}$. We define $\Gamma^{(\eta, Y)} f$ as the $\mathbb{C}^{n}$ - valued holomorphic function whose part in $\mathbb{C}^{d_{\ell}}$ is given by

$$
\left(\Gamma^{(\eta, Y)} f\right)_{\ell}=\sum_{j=0}^{\ell} \frac{1}{(\ell-j) !} \frac{1}{(2 \eta+2 j)_{\ell-j}} Y_{\ell} \cdots Y_{j+1} f_{j}^{(\ell-j)}
$$


for $\ell \geq j$. For invertible block diagonal $N$ on $\mathbb{C}^{n}$, we also define $\Gamma_{N}^{(\eta, Y)}:=\Gamma^{(\eta, Y)} \circ N$. It can be verified that $\Gamma_{N}^{(\eta, Y)}$ is a $\tilde{G}$ - equivariant isomorphism of $\mathbf{A}^{(\eta)}$ as a homogeneous holomorphic vector bundle onto $E^{(\eta, Y)}$. The image $K_{N}^{(\eta, Y)}$ of the reproducing kernel of $\mathbf{A}^{(\eta)}$ is then a reproducing kernel for $E^{(\eta, Y)}$. A computation gives that $K_{N}^{(\eta, Y)}(0,0)$ is a block diagonal matrix such that its $\ell$ 'th block is

$$
K_{N}^{(\eta, Y)}(0,0)_{\ell, \ell}=\sum_{j=0}^{\ell} \frac{1}{(\ell-j) !} \frac{1}{(2 \eta+2 j)_{\ell-j}} Y_{\ell} \cdots Y_{j+1} N_{j} N_{j}^{*} Y_{j+1}^{*} \cdots Y_{\ell}^{*} .
$$

We set

$$
H_{N}^{(\eta, Y)}=K_{N}^{(\eta, Y)}(0,0)^{-1} .
$$

We have now constructed a family $\left(E^{(\eta, Y)}, H_{N}^{(\eta, Y)}\right)$ of elementary homogeneous holomorphic vector bundles with a reproducing kernel ( $\eta>0, Y$ as before, $N$ invertible block diagonal).

Theorem 2.10. ([44, Theorem 3.2] Every elementary homogeneous holomorphic vector bundle E with a reproducing kernel arises from the construction given above.

Proof. (Sketch of proof) As a homogeneous bundle $E$ is isomorphic to some $E^{(\eta, Y)}$. Its reproducing kernel gives a Hilbert space structure in which the $\tilde{G}$ action on the sections of $E^{(\eta, Y)}$ is a unitary representation $U$. Now $\Gamma^{(\eta, Y)}$ intertwines the unitary representation of $\tilde{G}$ on $\mathbb{A}^{(\eta)}$ with $U$. The existence of a block diagonal $N$ such that $\Gamma_{N}^{(\eta, Y)}=\Gamma^{(\eta, Y)} \circ N$ is a Hilbert space isometry follows from Schur's Lemma.

As remarked before, every homogeneous holomorphic Hermitian vector bundle is isomorphic to an $\left(E^{(\eta, Y)}, I\right)$, here $Y$ is unique up to conjugation by a block unitary. In this form, it is easy to tell whether the bundle is irreducible: this is the case if and only if $Y$ is not the orthogonal direct sum of two matrices of the same block type as $Y$. We call such a $Y$ irreducible.

Let $\mathscr{P}$ be the set of all $(\eta, Y)$ such that $E^{(\eta, Y)}$ has a reproducing kernel. Using the formula for $K_{N}^{(\eta, Y)}(0,0)$ we can write down explicit systems of inequalities that determine whether $(\eta, Y)$ is in $\mathscr{P}$. In particular we have

Proposition 2.3. For every $Y$, there exists a $\eta_{Y}>0$ such that $(\eta, Y)$ is in $\mathscr{P}$ if and only if $\eta>\eta_{Y}$.

Finally, we obtain the desired classification.

Theorem 2.11. ([44, Theorem 4.1]) All the homogeneous holomorphic Hermitian vector bundles of rank $n$ with a reproducing kernel correspond to homogeneous operators in the Cowen - Douglas class $\mathrm{B}_{n}(\mathbb{D})$. The irreducible ones are the adjoint of the multiplication operator $M$ on the Hilbert space of sections of $\left(E^{(\eta, Y)}, I\right)$ for some $(\eta, Y)$ in $\mathscr{P}$ and irreducible $Y$. The block matrix $Y$ is determined up to conjugacy by block diagonal unitaries.

Proof. (Sketch of proof) There is a simple orthonormal system for the Hilbert space $\mathbb{A}^{(\lambda)}$. Hence we can find such a system for $\mathbf{A}^{(\eta)}$ as well. Transplant it using $\Gamma^{(\eta, Y)}$ to $E^{(\eta, Y)}$. The multiplication operator in this basis has a block diagonal form with

$$
M_{n}:=M_{\mid \operatorname{res}} \not{H}(n): \mathscr{H}(n) \rightarrow \mathscr{H}(n+1) .
$$

This description is sufficiently explicit to see:

$$
M_{n} \sim I+0\left(\frac{1}{n}\right) .
$$

Hence $M$ is the sum of an ordinary block shift operator and a Hilbert Schmidt operator. This completes the proof. 
The general case. The first examples of homogeneous operators in several variables were given in [52]. This was followed by a detailed study of these operators in the paper [5] for tube domains and in full generality in the paper [3]. First examples of homogeneous operators in $B_{2}(\mathbb{D})$ were given in [60]. A class of homogeneous operators in $B_{n}(\mathbb{D})$, which we called, generalized Wilkins operators were described in [6] using the jet construction. The paper [54] gives a class of homogeneous operators in $B_{n}(\mathscr{D})$, where $\mathscr{D}$ is one of the classical bounded symmetric domains, using the decomposition of a tensor product of two discrete series representations.

The essential ingredients from the case of the automorphism group of the unit disc is now available for an arbitrary bounded symmetric domain. In particular, the intertwining operator $\Gamma$ has been found explicitly. This gives a complete classification of homogeneous operators in the Cowen-Douglas class of the ball. In general, while a description of the homogeneous holomorphic vector bundles is given in the paper [46], it hasn't been possible to describe the operators as explicitly as in the case of the ball. These results were announced in [45] and complete proofs now appear in [46]. A different approach to finding a class of homogeneous holomorphic vector bundles is in [53].

As one might expect several questions remain open, for instance, which commuting tuple of homogeneous operators are subnormal, which of them induce a contractive, or completely contractive homomorphism. Some of these questions have been studied in [5, 3]. In a different direction, the class of quasi-homogeneous operators introduced in [39] containing all the homogeneous operators shares many of the properties of the smaller class of homogeneous operator. The Halmos question on similarity of polynomially bounded operators to a contraction has an affirmative answer for the quasi-homogeneous operators.

2.4. Quotient and sub-modules. In an attempt to generalize the very successful model theory of Sz.-Nagy and Foias for contractions to other settings, R. G. Douglas reformulated the question in the language of Hilbert modules over a function algebra. We describe below some aspects of this reformulation and its consequences focussing on the quotient modules for the class of quasi-free Hilbert modules $\mathscr{M}$ introduced in [26]. These Hilbert modules are obtained by taking the completion of the polynomial ring $\mathbb{C}[z]$ with respect to some inner product. We consider a class of sub-modules $\mathscr{S}_{k} \subseteq \mathscr{M}$ which consist of all functions in $\mathcal{M}$ that vanish to some fixed order $k$ on a hypersurface $\mathcal{Z}$ contained in $\Omega$. Let us recall some of the definitions (cf. [27, Section 1.2]).

(1) A hypersurface is a complex sub-manifold of complex dimension $m-1$, that is, a subset $\mathcal{Z} \subseteq \Omega$ is a hypersurface if for any fixed $z \in \mathcal{Z}$, there exists a neighbourhood $U \subseteq \Omega$ of $z$ and a local defining function $\varphi$ for $U \cap \mathcal{Z}$.

(2) A local defining function $\varphi$ is a holomorphic map $\varphi: U \rightarrow \mathbb{C}$ such that $U \cap \mathcal{Z}=\{z \in U: \varphi(z)=0\}$ and $\frac{f}{\varphi}$ is holomorphic on $U$ whenever $f_{\mid U \cap \mathscr{Z}}=0$. In particular, this implies that the gradient of $\varphi$ doesn't vanish on $\mathcal{Z}$ and that any two defining functions for $\mathcal{Z}$ must differ by a unit.

(3) A function $f$ is said to be vanishing to order $k$ on the hypersurface $\mathcal{Z}$ if $f=\varphi^{n} g$ for some $n \geq k$, a holomorphic function $g$ on $U$ and a defining function $\varphi$ of $\mathcal{Z}$. The order of vanishing on $\mathcal{Z}$ of a holomorphic function $f: \Omega \rightarrow \mathbb{C}$ does not depend on the choice of the local defining function. This definition can also be framed in terms of the partial derivatives normal to $\mathcal{Z}$.

We have seen that an extension of a result due to Aronszajn's provides a model for the quotient module when the sub-module consist of the maximal set of all functions vanishing on a hyper-surface. However, if the sub-module is taken to be all functions vanishing to order $k>1$, then the situation is different and one must introduce a matrix valued kernel via the jet construction.

2.4.1. The jet construction. Let $\mathscr{S}_{k} \subseteq \mathcal{M}$ be a sub-module of a quasi-free Hilbert module over the algebra $\mathscr{A}(\Omega)$ consisting of functions vanishing to order $k$ on a hyper-surface $\mathcal{Z} \subseteq \Omega$. Let $\partial$ denote the differentiation 
along the unit normal to the hyper-surface $\mathcal{Z}$. Let $J: \mathscr{M} \rightarrow \mathcal{M} \otimes \mathbb{C}^{k}$ defined by

$$
h \mapsto\left(h, \partial h, \partial^{2} h, \ldots, \partial^{k-1} h\right), h \in \mathscr{M}
$$

be the jet of order $k$. Transplanting the inner product from $\mathscr{M}$ on $\mathscr{M} \otimes \mathbb{C}^{k}$ via the map $J$, we see that

$$
\left\{\left(e_{n}, \partial e_{n}, \ldots, \partial^{k-1} e_{n}\right)_{n \geq 0}:\left(e_{n}\right)_{n \geq 0} \text { is an orthonormal basis in } \mathscr{M}\right\}
$$

is an orthonormal basis in $J(\mathscr{M}) \subseteq \mathscr{M} \otimes \mathbb{C}^{k}$. This makes the jet map $J$ isometric. Now, it is not hard to see that the sub-module $J \mathscr{S}_{k} \subseteq J \mathscr{M}$ consisting of the maximal set of functions in $J \mathscr{M}$ vanishing on $\mathcal{Z}$ is exactly the image under the map $J$ of the sub-module $\mathscr{S}_{k} \subseteq \mathscr{M}$. It is shown in [28] that the quotient module $\mathscr{Q}=\mathscr{M} / \mathscr{S}_{k}$ is isomorphic to $(J \mathscr{M}) /\left(J \mathscr{S}_{k}\right)$. Thus we are reduced to the multiplicity free case and it follows that the quotient module $\mathscr{Q}$ is the restriction of $J \mathscr{M}$ to the hypersurface $\mathcal{Z}$. To complete the description, we must provide a model.

The module $J \mathscr{M}$ possesses a reproducing kernel $J K$, which is the infinite sum

$$
J K(z, w)=\sum_{n=0}^{\infty}\left(J e_{n}\right)(z)\left(J e_{n}\right)(w)^{*}, z, w \in \Omega .
$$

From this it follows that $J K: \Omega \times \Omega \rightarrow \mathscr{M}_{k}(\mathbb{C})$ is of the form

$$
(J K)_{\ell, j}(z, w)=\left(\partial^{\ell} \bar{\partial}^{j} K\right)(z, w), 0 \leq \ell, j \leq k-1 .
$$

The module multiplication on $J \mathscr{M}$ is then naturally obtained by requiring that the map $J$ be a module map. Thus setting $J f$ to be the array

$$
(J f)_{\ell, j}=\left\{\begin{array}{ll}
\left(\begin{array}{l}
\ell \\
j
\end{array}\right)\left(\partial^{\ell-j} f\right), & 0 \leq \ell \leq j \leq k-1 \\
0 & \text { otherwise, }
\end{array},\right.
$$

$f \in \mathscr{A}(\Omega)$. The module multiplication is induced by the multiplication operator $M_{J_{f}}$, where $J_{f}$ is a holomorphic function defined on $\Omega$ taking values in $\mathscr{M}_{k}(\mathbb{C})$. The adjoint of this operator is easy to compute

$$
J_{f}^{*} J K(\cdot, w) \cdot \boldsymbol{x}=J K(\cdot, w)(J f)(w)^{*} \cdot \boldsymbol{x}, \boldsymbol{x} \in \mathbb{C}^{k} .
$$

We consider the Hilbert space $(J \mathscr{M})_{\text {res }}$ obtained by restricting the functions in $J \mathscr{M}$ to the hyper-surface $\mathcal{Z}$, that is,

$$
(J \mathscr{M})_{\text {res }}=\left\{\boldsymbol{h}_{0} \text { holomorphic on } \mathcal{Z}: \boldsymbol{h}_{0}=\boldsymbol{h}_{\mid Z} \text { for some } \boldsymbol{h} \in J \mathscr{M}\right\} .
$$

The norm of $\boldsymbol{h}_{0}$ in $(J \mathscr{M})_{\text {res }}$ is the quotient norm, that is,

$$
\left\|\boldsymbol{h}_{0}\right\|=\inf \left\{\|\boldsymbol{h}\|: \boldsymbol{h}_{\mid \mathcal{Z}}=\boldsymbol{h}_{0} \text { for } \boldsymbol{h} \in J \mathscr{M}\right\},
$$

and the module action is obtained by restricting the map $(f, \boldsymbol{h}) \rightarrow\left(J_{f}\right)_{\mid Z} \boldsymbol{h}_{\mid \mathcal{Z}}$.

We have discussed the restriction map $R$ before and observed that it is a unitary module map in the case of Hilbert modules consisting of scalar valued holomorphic functions. However, this is true of the vector valued case as well. It shows that $J K(\cdot, w)_{\text {res }}:=K(\cdot, w)_{\mid \mathcal{Z}}, w \in \mathcal{Z}$ is the kernel function for the Hilbert module $(J \mathscr{M})_{\text {res }}$.

Theorem 2.12. ([28, Theorem 3.3]) Let $\mathscr{M}$ be a Hilbert module over the algebra $\mathscr{A}(\Omega)$. If $\mathscr{S}_{k}$ is the sub-module of functions vanishing to order $k$, then the quotient module is isomorphic to $(J \mathscr{M})_{\mathrm{res}}$ via the isometric module map $J R$.

Finding invariants for the quotient module, except in the case of $k=1$, is more challenging. The module multiplication in the quotient module involves both a semi-simple and a nilpotent part. The semi-simple part lies typically in some $B_{n}(\mathcal{Z})$. Now, any equivalence between two quotient modules must also intertwine the nilpotent action. In the papers [25, 27], using this additional structure, a complete invariants were found 
for a class of quotient modules. We describe some fascinating possibilities for finding invariant for quotient modules using the notion of module tensor products and the recent work of Harvey and Lawson.

Let $\mathscr{M}$ and $\mathscr{N}$ be any two Hilbert modules over the algebra $\mathscr{A}$. Notice that there are two possible module actions on $\mathscr{M} \otimes \mathscr{N}$, i.e., the left action: $L \otimes I:(f, h \otimes k) \mapsto f \cdot h \otimes k$ and the right action: $I \otimes R:(f, h \otimes k) \mapsto$ $h \otimes f \cdot k$. The module tensor product $\mathscr{M} \otimes \mathscr{A} \mathscr{N}$ is defined to be the module obtained by identifying these two actions. Specifically, let $\mathscr{S}$ be the closed subspace of $\mathscr{M} \otimes \mathscr{N}$ generated by vectors of the form

$$
\{f \cdot h \otimes k-h \otimes f \cdot k: h \in \mathscr{M}, k \in \mathscr{N} \text { and } f \in \mathscr{A}\} .
$$

Then $\mathscr{S}$ is a submodule of $\mathscr{M} \otimes \mathscr{N}$ with respect to both the left and the right actions. The module tensor product $\mathscr{M} \otimes \mathscr{A} \mathscr{N}$ is defined to be $(\mathscr{M} \otimes \mathscr{N}) / \mathscr{S}$ together with the compression of either the left or the right actions, which coincide on this space. For fixed $w \in \Omega, \mathbb{C}$ is a module over $\mathscr{A}$ with the module action

$$
(f, v) \mapsto f(w) v, f \in \mathscr{A}, v \in \mathbb{C} .
$$

Let $\mathbb{C}_{w}$ denote the one dimensional module $\mathbb{C}$ with this action. We will largely confine ourselves to the module tensor product $\mathscr{M} \otimes_{\mathscr{A}} \mathbb{C}_{w}$, which we denote by $\mathscr{M}(w)$.

Localizing the short exact sequence

$$
0 \longleftarrow \mathscr{Q} \longleftarrow \mathscr{M} \stackrel{X}{\longleftarrow} \mathscr{S} \longleftarrow 0
$$

using the one dimensional module $\mathbb{C}_{w}$, one obtains a new short exact sequence of spectral sheaves (cf. [29])

$$
0 \longleftarrow \mathscr{Q} \otimes_{\mathscr{A}(\Omega)} \mathbb{C}_{w} \longleftarrow \mathscr{M} \otimes_{\mathscr{A}(\Omega)} \mathbb{C}_{w} \stackrel{X \otimes_{\mathscr{A}(\Omega)}}{\longleftarrow} \mathbb{1}_{w} \mathscr{S} \otimes_{\mathscr{A}(\Omega)} \mathbb{C}_{w} \longleftarrow 0 .
$$

Let $E_{0}$ and $E$ be the holomorphic line bundles corresponding to the modules $\mathscr{S}$ and $\mathscr{M}$ and $\mathscr{K}_{E_{0}}, \mathscr{K}_{E}$ be their curvatures, respectively. It is shown in [24] that the class of the alternating sum

$$
\sum_{i, j=1}^{m} \frac{\partial^{2}}{\partial w_{i} \partial \bar{w}_{j}} \log \left(X(w)^{*} X(w)\right) d w_{i} \wedge d \bar{w}_{j}-\mathscr{K}_{E_{0}}(w)+\mathscr{K}_{E}(w),
$$

is the fundamental class of the hypersurface $\mathcal{Z}$. This identification makes essential use of the PoincaréLelong formula.

It is not clear how one can obtain such an alternating sum if the submodule $\mathscr{S}$ is not assumed to be the submodule (maximal set) of functions vanshing on $\mathcal{Z}$. A possible approach to this question using some ideas of Doanldson appearing in [22] is discussed in [28]. An adaptation of the results of Harvey and Lawson [35] to the present situation may be fruitful in the case where $\mathscr{S}$ is assumed to consist of all functions in $\mathscr{M}$ which vanish to order $k$. Let $\mathscr{E}$ and $\mathscr{E}_{0}$ be the vector bundles obtained by localization (cf. [29]) from the modules $\mathscr{M}$ and $\mathscr{M}_{0}$ and let $\phi$ be an ad-invariant polynomial (in particular, a Chern form) in the respective curvatures $\mathscr{K}$ and $\mathscr{K}_{0}$. Then the work of Harvey and Lawson [35] on singular connections gives a mechanism for studying these bundles since the natural connection on the bundle $\mathscr{M}_{0}$ is a singular one. They obtain a relation of the form

$$
\phi(\mathscr{K})-\phi\left(\mathscr{K}_{0}\right)=\operatorname{Res}_{\phi}[\mathcal{Z}]+d T_{\phi},
$$

where $\operatorname{Res}_{\phi}[\mathfrak{Z}]$ is a 'residue' form related to the zero set and $T_{\phi}$ is a transgression current. This incorporates a generalized Poincaré-Lelong formula which played a crucial role in the study of the quotient module in the rank one case, see [28].

2.4.2. The Clebsch-Gorden formula. Let $\mathscr{M}_{1}$ and $\mathscr{M}_{2}$ be Hilbert spaces consisting of of holomorphic functions defined on $\Omega$ possessing reproducing kernels $K_{1}$ and $K_{2}$, respectively. Assume that the natural action of $\mathbb{C}[z]$ on the Hilbert space $\mathscr{M}_{1}$ is continuous, that is, the map $(p, h) \rightarrow p h$ defines a bounded operator on 
$\mathscr{M}$ for $p \in \mathbb{C}[z]$. (We make no such assumption about the Hilbert space $\mathscr{M}_{2}$.) Now, $\mathbb{C}[z]$ acts naturally on the Hilbert space tensorproduct $\mathscr{M}_{1} \otimes \mathscr{M}_{2}$ via the map

$$
(p,(h \otimes k)) \rightarrow p h \otimes k, p \in \mathbb{C}[z], h \in \mathscr{M}_{1}, k \in \mathscr{M}_{2} .
$$

The map $h \otimes k \rightarrow h k$ identifies the Hilbert space $\mathscr{M}_{1} \otimes \mathscr{M}_{2}$ as a reproducing kernel Hilbert space of holomorphic functions on $\Omega \times \Omega$. The module action is then the point-wise multiplication $(p, h k) \rightarrow(p h) k$, where

$$
((p h) k)\left(z_{1}, z_{2}\right)=p\left(z_{1}\right) h\left(z_{1}\right) k\left(z_{2}\right), \quad z_{1}, z_{2} \in \Omega .
$$

Let $\mathscr{M}$ be the Hilbert module $\mathscr{M}_{1} \otimes \mathscr{M}_{2}$ over $\mathbb{C}[z]$. Let $\triangle \subseteq \Omega \times \Omega$ be the diagonal subset $\{(z, z): z \in \Omega\}$ of $\Omega \times \Omega$. Let $\mathscr{S}$ be the maximal submodule of functions in $\mathscr{M}_{1} \otimes \mathscr{M}_{2}$ which vanish on $\triangle$. Thus

$$
0 \rightarrow \mathscr{S} \stackrel{X}{\rightarrow} \mathscr{M}_{1} \otimes \mathscr{M}_{2} \stackrel{Y}{\rightarrow} \mathscr{Q} \rightarrow 0
$$

is a short exact sequence, where $\mathscr{Q}=\left(\mathscr{M}_{1} \otimes \mathscr{M}_{2}\right) / \mathscr{S}, X$ is the inclusion map and $Y$ is the natural quotient map.

As we have seen earlier in Section 1.4, the theorem of Aronszajn provides a complete description of the quotient module $\mathscr{Q}$ as the restrictions of functions in $\mathscr{M}$. Now, let us investigate what happens if the submodule $\mathscr{S}$ is taken to be space of functions vanishing to order 2 on $\triangle$. Set $\mathscr{H}_{1}$ and $\mathscr{H}_{2}$ to be the submodules defined by

$$
\mathscr{H}_{1}=\left\{f \in(\mathscr{H}, K) \otimes(\mathscr{H}, K):\left.f\right|_{\Delta}=0\right\}
$$

and

$$
\mathscr{H}_{2}=\left\{f \in(\mathscr{H}, K) \otimes(\mathscr{H}, K):\left.f\right|_{\Delta}=\left.\partial_{1} f\right|_{\Delta}=\left.\partial_{2} f\right|_{\Delta}=\ldots=\left.\partial_{m} f\right|_{\Delta}=0\right\} .
$$

Let $\mathscr{H}_{11}=\mathscr{H}_{2}^{\perp} \ominus \mathscr{H}_{1}^{\perp}$ We have already described the quotient module $\mathscr{H}_{00}:=\mathscr{M} \ominus \mathscr{H}_{1}$, where $\mathscr{M}=(\mathscr{H}, K) \otimes$ $(\mathscr{H}, K)$. This is the module $\mathscr{M}_{\text {res. }}$ Set

$$
\widetilde{K}(z, w)=\left(K^{2}(z, w) \partial_{i} \bar{\partial}_{j} \log K(z, w)\right)_{1 \leq i, j \leq m} .
$$

We claim that the function $\tilde{K}$ taking values in $\mathscr{M}_{m}(\mathbb{C})$ is a non-negative definite kernel. To see this, set

$$
\phi_{i}(w):=K_{w} \otimes \bar{\partial}_{i} K_{w}-\bar{\partial}_{i} K_{w} \otimes K_{w}, \quad 1 \leq i \leq m,
$$

and note that each $\phi_{i}: \Omega \rightarrow \mathscr{M}$ is holomorphic. A simple calculation then shows that

$$
\left(\left(\left\langle\phi_{j}(w), \phi_{i}(z)\right\rangle_{M}\right)\right)=\widetilde{K}(z, w) .
$$

How to describe the Hilbert space, or more importantly, the Hilbert module $(\mathscr{H}, \widetilde{K})$ ? May be, it is a quotient, or a sub-quotient module, of the Hilbert module $(\mathscr{H}, K) \otimes(\mathscr{H}, K)$ ?

Let $\mathscr{H}_{0}$ be the subspace of $(\mathscr{H}, K) \otimes(\mathscr{H}, K)$ given by the smallest closed subspace containing the linear span of the vectors $\left\{\phi_{i}(w): w \in \Omega, 1 \leq i \leq m\right\}$. From this definition, it is not clear which functions belong to the subspace. An interesting limit computation given below shows that it coincides with $\mathscr{H}_{11}$.

The point of what we have said so far is that we can explicitly describe the Hilbert modules $\mathscr{H}_{2}^{\perp}$ and $\mathscr{H}_{1}^{\perp}$, up to an isomorphism of modules. Using the jet construction followed by the restriction map, one may also describe the direct sum $\mathscr{H}_{2}^{\perp} \oplus \mathscr{H}_{1}^{\perp}$, again up to an isomorphism.

But what is the module $\mathscr{H}_{11}$ ? To answer this question (see [33, Section 2.4.1]), one must find the kernel function for $\mathscr{H}_{11}$. Setting $K_{1}$ to be the kernel function of the module $\mathscr{H}_{1}$, indeed, we have

$$
\lim _{u \rightarrow z, v \rightarrow w} \frac{K_{1}(z, u ; v, w)}{(z-u)(\bar{w}-\bar{v})}=\frac{1}{2} K(z, w)^{2} \partial \bar{\partial} \log K(z, w) .
$$

This shows that $\mathscr{H}_{11}$ is isomorphic to $(\mathscr{H}, \tilde{K})$. The main challenge is to obtain an orthogonal decomposition of the Hilbert module $\mathcal{M}$ in the form of a composition series, namely, to complete the decomposition:

$$
\mathscr{H}_{31} \sim \mathscr{H}_{00} \oplus \mathscr{H}_{11} \oplus \mathscr{H}_{22} \oplus \cdots,
$$


where $\mathscr{H}_{00}$ is the quotient module $\mathscr{M}_{\text {res }}$, as in the more familiar Clebsch-Gorden formula.

2.4.3. The sheaf model. For a Hilbert module $\mathscr{M}$ over a function algebra $\mathscr{A}(\Omega)$, not necessarily in the class $\mathrm{B}_{1}(\Omega)$, motivated by the correspondence of vector bundles with locally free sheaf, we construct a sheaf of modules $\mathscr{S}^{\mathscr{M}}(\Omega)$ over $\mathscr{O}(\Omega)$ corresponding to $\mathcal{M}$. We assume that $\mathscr{M}$ possesses all the properties for it to be in the class $\mathrm{B}_{1}(\Omega)$ except that the dimension of the joint kernel $\mathbb{K}(w)$ need not be constant. We note that sheaf models have occured, as a very useful tool, in the study of analytic Hilbert modules (cf. [31]). Although, the model we describe below is somewhat different.

A Hilbert module $\mathscr{M} \subset \mathscr{O}(\Omega)$ is said to be in the class $\mathfrak{B}_{1}(\Omega)$ if it possesses a reproducing kernel $K$ (we don't rule out the possibility: $K(w, w)=0$ for $w$ in some closed subset $X$ of $\Omega$ ) and the dimension of $\mathscr{M} / \mathfrak{m}_{w} \mathscr{M}$ is finite for all $w \in \Omega$.

Most of the examples in $\mathfrak{B}_{1}(\Omega)$ arises in the form of a submodule of some Hilbert module $\mathscr{H}(\subseteq \mathscr{O}(\Omega)$ ) in the Cowen-Douglas class $B_{1}(\Omega)$. Are there others?

Let $\mathscr{S}^{\mathscr{M}}(\Omega)$ be the subsheaf of the sheaf of holomorphic functions $\mathscr{O}(\Omega)$ whose stalk at $w \in \Omega$ is

$$
\left\{\left(f_{1}\right)_{w} \mathscr{O}_{w}+\cdots+\left(f_{n}\right)_{w} \mathscr{O}_{w}: f_{1}, \ldots, f_{n} \in \mathcal{M}\right\}
$$

or equivalently,

$$
\mathscr{S}^{\mathscr{M}}(U)=\left\{\sum_{i=1}^{n}\left(f_{i \mid U}\right) g_{i}: f_{i} \in \mathscr{M}, g_{i} \in \mathscr{O}(U)\right\}
$$

for $U$ open in $\Omega$.

Proposition 2.4. The sheaf $\mathscr{S}^{\mathscr{M}}(\Omega)$ is coherent.

Proof. The sheaf $\mathscr{S}^{\mathscr{M}}(\Omega)$ is generated by the set of functions $\{f: f \in \mathscr{M}\}$. Let $\mathscr{S}_{J}^{\mathscr{M}}(\Omega)$ be the subsheaf generated by the set of functions

$$
J=\left\{f_{1}, \ldots, f_{\ell}\right\} \subseteq \mathscr{M} \subseteq \mathscr{O}(\Omega) .
$$

Thus $\mathscr{S}_{J}^{M}(\Omega)$ is coherent. An application of Noether's Lemma [34] then guarantees that

$$
\mathscr{S}^{\mathscr{M}}(\Omega)=\cup_{J \text { finite }} \mathscr{S}_{J}^{\mathscr{M}}(\Omega)
$$

is coherent.

We note that the coherence of the sheaf implies, in particular, that the stalk $\left(\mathscr{S}^{\mathscr{M}}\right)_{w}$ at $w \in \Omega$ is generated by a finite number of elements $g_{1}, \ldots, g_{n}$ from $\mathscr{O}(\Omega)$.

If $K$ is the reproducing kernel for $\mathscr{M}$ and $w_{0} \in \Omega$ is a fixed but arbitrary point, then for $w$ in a small neighborhood $U$ of $w_{0}$, we obtain the following decomposition theorem.

Theorem 2.13. Suppose $g_{i}^{0}, 1 \leq i \leq n$, be a minimal set of generators for the stalk $\left(\mathscr{S}^{\mathscr{M}}\right)_{0}:=\left(\mathscr{S}^{\mathscr{M}}\right)_{w_{0}}$. Then we have

$$
K(\cdot, w):=K_{w}=g_{1}^{0}(w) K_{w}^{(1)}+\cdots+g_{n}^{0}(w) K_{w}^{(n)},
$$

where $K^{(p)}: U \rightarrow \mathcal{M}$ defined by $w \mapsto K_{w}^{(p)}, 1 \leq p \leq n$, is anti-holomorphic. Moreover, the elements $K_{w_{0}}^{(p)}, 1 \leq$ $p \leq n$ are linearly independent in $\mathcal{M}$, they are eigenvectors for the adjoint of the action of $\mathscr{A}(\Omega)$ on the Hilbert module $\mathcal{M}$ at $w_{0}$ and are uniquely determined by these generators.

We also point out that the Grammian

$$
G(w)=\left(\left(\left\langle K_{w}^{(p)}, K_{w}^{(q)}\right\rangle\right)\right)_{p, q=1}^{n}
$$

is invertible in a small neighborhood of $w_{0}$ and is independent of the generators $g_{1}, \ldots, g_{n}$. Thus

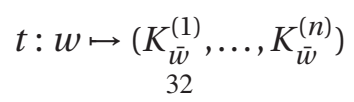


defines a holomorphic map into the Grassmannian $\mathrm{G}(\mathscr{H}, n)$ on the open set $U^{*}$. The pull-back $E_{0}$ of the canonical bundle on $\mathrm{G}(\mathscr{H}, n)$ under this map then define a holomorphic Hermitian bundle on $U^{*}$. Clearly, the decomposition of $K$ given in our Theorem is not canonical in anyway. So, we can't expect the corresponding vector bundle $E_{0}$ to reflect the properties of the Hilbert module $\mathscr{M}$. However, it is possible to obtain a canonical decomposition following the construction in [21]. It then turns out that the equivalence class of the corresponding vector bundle $E_{0}$ obtained from this canonical decomposition is an invariant for the isomorphism class of the Hilbert module $\mathscr{M}$. These invariants are by no means easy to compute. At the end of this subsection, we indicate, how to construct invariants which are more easily computable. For now, the following Corollary to the decomposition theorem is immediate.

Corollary 2.2. The dimension of the joint kernel $\mathbb{K}(w)$ is greater or equal to the number of minimal generators of the stalk $\left(\mathscr{S}^{\mathscr{M}}\right)_{w}$ at $w \in \Omega$.

Now is the appropriate time to raise a basic question. Let $\mathfrak{m}_{w} \subseteq \mathscr{A}(\Omega)$ be the maximal ideal of functions vanishing at $w$. Since we have assumed $\mathfrak{m}_{w} \mathscr{M}$ is closed, it follows that the dimension of the joint kernel $\mathbb{K}(w)$ equals the dimension of the quotient module $\mathscr{M} /\left(\mathfrak{m}_{w} \mathscr{M}\right)$. However it is not clear if one may impose natural hypothesis on $\mathscr{M}$ to ensure

$$
\operatorname{dim} \mathscr{M} /\left(\mathfrak{m}_{w} \mathscr{M}\right)=\operatorname{dim} \mathbb{K}(w)=\operatorname{dim}\left(\mathscr{S}^{\mathscr{M}}\right)_{w} /\left(\mathfrak{m}\left(\mathscr{O}_{w}\right)\left(\mathscr{S}^{\mathscr{M}}\right)_{w}\right),
$$

where $\mathfrak{m}\left(\mathscr{O}_{w}\right)$ is the maximal ideal in $\mathscr{O}_{w}$, as well.

More generally, suppose $p_{1}, \ldots, p_{n}$ generate $\mathscr{M}$. Then $\operatorname{dim} \mathbb{K}(w) \leq n$ for all $w \in \Omega$. If the common zero set $V$ of these is $\{0\}$ then $\left(p_{1}\right)_{0}, \ldots,\left(p_{n}\right)_{0}$ need not be a minimal set of generators for $\left(\mathscr{S}^{\mathcal{M}}\right)_{0}$. However, we show that they do if we assume $p_{1}, \ldots, p_{n}$ are homogeneous of degree $k$, say. Further more, basis for $\mathbb{K}(0)$ is the set of vectors:

where $\overline{\boldsymbol{\partial}}=\left(\bar{\partial}_{1}, \ldots, \bar{\partial}_{m}\right)$.

$$
\left.\left.\left\{p_{1}(\overline{\boldsymbol{\partial}})\right\} K(\cdot, w)_{\mid w=0}, \ldots, p_{n}(\overline{\boldsymbol{\partial}})\right\} K(\cdot, w)_{\mid w=0}\right\},
$$

Going back to the example of $H_{0}^{2}\left(\mathbb{D}^{2}\right)$, we see that it has two generators, namely $z_{1}$ and $z_{2}$. Clearly, the joint kernel

at $w=\left(w_{1}, w_{2}\right)$ is spanned by

$$
\mathbb{K}(w):=\operatorname{ker} D_{\left(M_{1}^{*}-\bar{w}_{1}, M_{2}^{*}-\bar{w}_{2}\right)}
$$

$$
\left\{z_{1} \otimes_{\mathscr{A}\left(\mathbb{D}^{2}\right)} 1_{w}, z_{2} \otimes_{\mathscr{A}\left(\mathbb{D}^{2}\right)} 1_{w}\right\}=\left\{w_{1} K_{H_{0}^{2}\left(\mathbb{D}^{2}\right)}(z, w), w_{2} K_{H_{0}^{2}\left(\mathbb{D}^{2}\right)}(z, w)\right\}
$$

which consists of two vectors that are linearly dependent except when $w=(0,0)$. We also easily verify that

$$
\left(\mathscr{S}^{H_{0}^{2}\left(\mathbb{D}^{2}\right)}\right)_{w} \cong \begin{cases}\mathscr{O}_{w} & w \neq(0,0) \\ \mathfrak{m}\left(\mathscr{O}_{0}\right) & w=(0,0) .\end{cases}
$$

Since the reproducing kernel

$$
K_{H_{0}^{2}\left(\mathbb{D}^{2}\right)}(z, w)=K_{H^{2}\left(\mathbb{D}^{2}\right)}(z, w)-1=\frac{z_{1} \bar{w}_{1}+z_{2} \bar{w}_{2}-z_{1} z_{2} \bar{w}_{1} \bar{w}_{2}}{\left(1-z_{1} \bar{w}_{1}\right)\left(1-z_{2} \bar{w}_{2}\right)},
$$

we find there are several choices for $K_{w}^{(1)}$ and $K_{w}^{(2)}, w \in U$. However, all of these choices disappear if we set $\bar{w}_{1} \theta_{1}=\bar{w}_{2}$ for $w_{1} \neq 0$, and take the limit:

$$
\lim _{\left(w_{1}, w_{2}\right) \rightarrow 0} \frac{K_{H_{0}^{2}\left(\mathbb{D}^{2}\right)}(z, w)}{\bar{w}_{1}}=K_{0}^{(1)}(z)+\theta_{1} K_{0}^{(2)}(z)=z_{1}+\theta_{1} z_{2}
$$

because $K_{0}^{(1)}$ and $K_{0}^{(2)}$ are uniquely deteremined by Theorem 1 . Similarly, for $\bar{w}_{2} \theta_{2}=\bar{w}_{1}$ for $w_{2} \neq 0$, we have

$$
\lim _{\left(w_{1}, w_{2}\right) \rightarrow 0} \frac{K_{H_{0}^{2}\left(\mathbb{D}^{2}\right)}(z, w)}{\bar{w}_{2}}=K_{0}^{(2)}(z)+\theta_{2} K_{0}^{(1)}(z)=z_{2}+\theta_{2} z_{1} .
$$


Thus we have a Hermitian line bundle on the complex projective space $\mathbb{P}^{1}$ given by the frame $\theta_{1} \mapsto z_{1}+\theta_{1} z_{2}$ and $\theta_{2} \mapsto z_{2}+\theta_{2} z_{1}$. The curvature of this line bundle is then an invariant for the Hilbert module $H_{0}^{2}\left(\mathbb{D}^{2}\right)$ as shown in [28]. This curvature is easily calculated and is given by the formula $\mathscr{K}(\theta)=\left(1+|\theta|^{2}\right)^{-2}$. The decomposition theorem yields similar results in many other examples.

Let $\mathscr{I}$ be an ideal in the polynomial ring $\mathbb{C}[\mathbf{z}]$. The characteristic space of an ideal $\mathscr{I}$ in $\mathbb{C}[\mathbf{z}]$ at the point $w$ is the vector space

$$
\mathbb{V}_{w}(\mathscr{I}):=\left\{q \in \mathbb{C}[\mathbf{z}]:\left.q(D) p\right|_{w}=0, p \in \mathscr{I}\right\} .
$$

The envolope $\mathscr{I}_{w}^{e}$ of the ideal $\mathscr{I}$ is

$$
\left\{p \in \mathbb{C}[\mathbf{z}]:\left.q(D) p\right|_{w}=0, q \in \mathbb{V}_{w}(\mathscr{I})\right\} .
$$

If the zero set of the ideal $\mathscr{I}$ is $\{w\}$ then $\mathscr{I}_{w}^{e}=\mathbb{V}_{w}(\mathscr{I})$.

This describes an ideal by prescribing conditions on derivatives. We stretch this a little more. Let $\tilde{\mathbb{V}}_{w}(\mathscr{I})$ be the auxiliary space $\mathbb{V}_{w}\left(\mathfrak{m}_{w} \mathscr{I}\right)$. We have

$$
\operatorname{dim}\left(\cap \operatorname{Ker}\left(M_{j}-w_{j}\right)^{*}\right)=\operatorname{dim} \tilde{\mathbb{V}}_{w}(\mathscr{I}) / \mathbb{V}_{w}(\mathscr{I}) .
$$

Actually, we have something much more substantial.

Lemma 2.1. Fix $w_{0} \in \Omega$ and polynomials $q_{1}, \ldots, q_{t}$. Let $\mathscr{I}$ be a polynomial ideal and $K$ be the reproducing kernel corresponding the Hilbert module $[\mathscr{I}]$, which is assumed to be in $\mathfrak{B}_{1}(\Omega)$. Then the vectors

$$
\left.q_{1}(\bar{D}) K(\cdot, w)\right|_{w=w_{0}}, \ldots,\left.q_{t}(\bar{D}) K(\cdot, w)\right|_{w=w_{0}}
$$

form a basis of the joint kernel $\cap_{j=1}^{m} \operatorname{ker}\left(M_{j}-w_{0 j}\right)^{*}$ if and only if the classes $\left[q_{1}^{*}\right], \ldots,\left[q_{t}^{*}\right]$ form a basis of $\tilde{\mathbb{V}}_{w_{0}}(\mathscr{I}) / \mathbb{V}_{w_{0}}(\mathscr{I})$.

However, it is not clear if we can choose the polynomials $\left\{q_{1}, \ldots, q_{t}\right\}$ to be a generating set for the ideal $\mathscr{I}$. None the less, the following theorem produces a new set $\left\{q_{1}, \ldots, q_{v}\right\}$ of generators for $\mathscr{I}$, which is more or less "canonical". Indeed, it is uniquely determined modulo linear transformations.

Theorem 2.14. ([10, Proposition 2.10]) Let $\mathscr{I} \subset \mathbb{C}[\mathbf{z}]$ be a homogeneous ideal and $\left\{p_{1}, \ldots, p_{\nu}\right\}$ be a minimal set of generators for $\mathscr{I}$ consisting of homogeneous polynomials. Let $K$ be the reproducing kernel corresponding to the Hilbert module $[\mathscr{I}]$, which is assumed to be in $\mathfrak{B}_{1}(\Omega)$. Then there exists a set of generators $q_{1}, \ldots, q_{v}$ for the ideal $\mathscr{I}$ such that the set

$$
\left\{\left.q_{i}(\bar{D}) K(\cdot, w)\right|_{w=0}: 1 \leq i \leq v\right\}
$$

is a basis for the joint kernel $\cap_{j=1}^{m} \operatorname{ker} M_{j}^{*}$.

2.5. The flag structure. We have already discussed the class of operators $\mathscr{F} B_{2}(\Omega)$ which is contained in the Cowen-Douglas class $B_{2}(\Omega)$. A natural generalization is given below. The main point of introducing this smaller class is to show that a complete set of tractable unitary invariants exist for this class.

Definition 2.1. We let $\mathscr{F} B_{n}(\Omega)$ be the set of all bounded linear operators $T$ defined on some complex separable Hilbert space $\mathscr{H}=\mathscr{H}_{0} \oplus \cdots \oplus \mathscr{H}_{n-1}$, which are of the form

$$
T=\left(\begin{array}{ccccc}
T_{0} & S_{0,1} & S_{0,2} & \ldots & S_{0, n-1} \\
0 & T_{1} & S_{1,2} & \cdots & S_{1, n-1} \\
\vdots & \ddots & \ddots & \ddots & \vdots \\
0 & \cdots & 0 & T_{n-2} & S_{n-2, n-1} \\
0 & \cdots & \cdots & 0 & T_{n-1}
\end{array}\right),
$$


where the operator $T_{i}: \mathscr{H}_{i} \rightarrow \mathscr{H}_{i}$, defined on the complex separable Hilbert space $\mathscr{H}_{i}, 0 \leq i \leq n-1$, is assumed to be in $B_{1}(\Omega)$ and $S_{i, i+1}: \mathscr{H}_{i+1} \rightarrow \mathscr{H}_{i}$, is assumed to be a non-zero intertwining operator, namely, $T_{i} S_{i, i+1}=S_{i, i+1} T_{i+1}, 0 \leq i \leq n-2$.

The set of operators in $\mathscr{F} B_{n}(\Omega)$, as before, also belong to the Cowen-Douglas class $B_{n}(\Omega)$. To show this starting with the base case of $n=2$, using induction, we don't even need the intertwining condition. A rigidity theorem for this class of operators was proved in [38, Theorem 3.5], which is reproduced below.

Theorem 2.15 (Rigidity). Any two operators $T$ and $\tilde{T}$ in $\mathscr{F} B_{n}(\Omega)$ are unitarily equivalent if and only if there exists unitary operators $U_{i}, 0 \leq i \leq n-1$, such that $U_{i} T_{i}=\tilde{T}_{i} U_{i}$ and $U_{i} S_{i, j}=\tilde{S}_{i, j} U_{j}, i<j$.

From the rigidity, it is easy to extract unitary invariants for the operators in the class $\mathscr{F} B_{n}(\Omega)$, see [38, Theorem 3.6]. These invariants come from the intrinsic complex geometry inherent in the definition of the operators in the class $\mathscr{F} B_{n}(\Omega)$. Also, all the operators in this class are irreducible.

2.5.1. An application to module tensor products. In the early development of the quotient and sub-modules, it was expected that the localization using modules of rank $>1$ might provide new insight. This was discussed in some detail in the paper [28] but the outcome was not conclusive. The results from [38, Section 4] completes the study initiated in [28]. Let $f$ be a polynomial in one variable. Set

$$
\mathscr{J}_{\boldsymbol{\mu}}(f)(z)=\left(\begin{array}{cccc}
\mu_{1,1} f(z) & 0 & \cdots & 0 \\
\mu_{2,1} \frac{\partial}{\partial z} f(z) & \mu_{2,2} f(z) & \cdots & 0 \\
\vdots & \vdots & \ddots & \vdots \\
\mu_{k, 1} \frac{\partial^{k-1}}{\partial z^{k-1}} f(z) & \mu_{k-1,1} \frac{\partial^{k-2}}{\partial z^{k-2}} f(z) & \cdots & \mu_{k, k} f(z)
\end{array}\right)
$$

where $\boldsymbol{\mu}=\left(\mu_{i, j}\right)$ is a lower triangular matrix of complex numbers with $\mu_{i, i}=1,1 \leq i \leq k$. Lemma 4.1 of [38] singles out those $\boldsymbol{\mu}$ which ensure $\mathscr{J}_{\boldsymbol{\mu}}(f g)=\mathscr{J}_{\boldsymbol{\mu}}(f) \mathscr{J}_{\boldsymbol{\mu}}(g)$. Fix one such $\boldsymbol{\mu}$. For $\mathbf{x}$ in $\mathbb{C}^{k}$, and $f$ in the polynomial ring $\mathbb{C}[z]$, define the module multiplication on $\mathbb{C}^{k}$ as follows:

$$
f \cdot \mathbf{x}=\mathscr{J}_{\boldsymbol{\mu}}(f)(w) \mathbf{x}
$$

The finite dimensional Hilbert space $\mathbb{C}^{k}$ equipped with this module multiplication is denoted by $\mathbb{C}_{w}^{k}[\boldsymbol{\mu}]$.

Let $\mathscr{M}$ be a Hilbert module possessing a reproducing kernel. We construct a module of $k$ - jets by setting

$$
J \mathscr{M}=\left\{\sum_{l=0}^{k-1} \frac{\partial^{i}}{\partial z^{i}} h \otimes \varepsilon_{i+1}: h \in \mathscr{M}\right\}
$$

where $\varepsilon_{i+1}, 0 \leq i \leq k-1$, are the standard basis vectors in $\mathbb{C}^{k}$. Recall that there is a natural module action on $J \mathscr{M}$, namely,

$$
\left(f, \sum_{l=0}^{k-1} \frac{\partial^{i}}{\partial z^{i}} h\right) \mapsto \mathscr{J}(f)\left(\sum_{l=0}^{k-1} \frac{\partial^{i}}{\partial z^{i}} h \otimes \varepsilon_{i+1}\right), f \in \mathbb{C}[z], h \in \mathscr{M}
$$

where

$$
\mathscr{J}(f)_{i, j}= \begin{cases}\left(\begin{array}{l}
i-1 \\
j-1
\end{array}\right) \partial^{i-j} f & \text { if } i \geq j, \\
0 & \text { otherwise. }\end{cases}
$$

The module tensor product $J \mathscr{M} \otimes_{\mathbb{C}[z]} \mathbb{C}_{w}^{k}[\boldsymbol{\mu}]$ is easily identified with the quotient module $\mathscr{N}^{\perp}$, where $\mathscr{N} \subseteq \mathscr{M}$ is the sub-module spanned by the vectors

$$
\left\{\sum_{l=1}^{k}\left(J_{f} \cdot \mathbf{h}_{l} \otimes \varepsilon_{l}-\mathbf{h}_{l} \otimes\left(\mathscr{J}_{\boldsymbol{\mu}}(f)\right)(w) \cdot \varepsilon_{l}\right): \mathbf{h}_{l} \in J \mathscr{M}, \varepsilon_{l} \in \mathbb{C}^{k}, f \in[z]\right\} .
$$


Theorem 2.16. The Hilbert modules corresponding to the localizations $J \mathscr{M} \otimes_{\mathbb{C}[z]} \mathbb{C}_{w}^{k}\left[\boldsymbol{\mu}_{i}\right], i=1,2$, are in $\mathscr{F} B_{k}(\Omega)$ and they are isomorphic if and only if $\boldsymbol{\mu}_{1}=\boldsymbol{\mu}_{2}$.

Acknowledgement. The author thanks Soumitra Ghara, Dinesh Kumar Keshari, Md. Ramiz Reza and Subrata Shyam Roy for going through a preliminary draft of this article carefully and pointing out several typographical errors. The author gratefully acknowledges the financial support from the Department of Science and Technology in the form of the J C Bose National Fellowship and from the University Grants Commission, Centre for Advanced Study.

\section{REFERENCES}

[1] M. B. Abrahamse and R. G. Douglas, A class of subnormal operators related to multiply connected domains, Adv. Math. (1976), $106-148$.

[2] O. P. Agrawal and N. Salinas, Sharp kernels and canonical subspaces (revised), Amer. J. Math. 110 (1988), 23 - 47.

[3] J. Arazy and G. Zhang, Homogeneous multiplication operators on bounded symmetric domains, J. Func. Anal. 202 (2003), $44-66$.

[4] N. Aronszajn, Theory of reproducing kernels, Trans. Amer. Math. Soc. 68 (1950), 337 - 404.

[5] B. Bagchi and G. Misra, Homogeneous tuples of multiplication operators on twisted Bergman space, J. Func. Anal. 136 (1996), $171-213$.

[6] Homogeneous operators and projective representations of the Möbius group: a survey, Proc. Ind. Acad. Sc.(Math. Sci.) 111 (2001), 415 - 437.

[7] I. Biswas and G. Misra, $\widetilde{\mathrm{SL}}(2, \mathbb{R})$-homogeneous vector bundles, Internat. J. Math. 19 (2008), 1 - 19.

[8] S. Biswas, G. Ghosh, G. Misra, and S. Shyam Roy, On reducing sub-modules of hilbert modules with $\mathfrak{S}_{n}$-invaraint kernels, preprint, 2017.

[9] S. Biswas, D. K. Keshari, and G. Misra, Infinitely divisible metrics and curvature inequalities for operators in the CowenDouglas class, J. Lond. Math. Soc. (2) 88 (2013), no. 3, 941 - 956.

[10] S. Biswas and G. Misra, Resolution of singularities for a class of Hilbert modules, Indiana Univ. Math. J. 61 (2012), 1019 1050.

[11] S. Biswas, G. Misra, and M. Putinar, Unitary invariants for Hilbert modules of finite rank, J. Reine Angew. Math. 662 (2012), $165-204$.

[12] Li Chen, A dual geometric theory for bundle shifts, J. Func. Anal. 263 (2012), 846 - 868.

[13] X. Chen and R. G. Douglas, Localization of Hilbert modules, Mich. Math. J. 39 (1992), 443 - 454.

[14] X. Chen and K. Guo, Analytic hilbert modules, Chapman and Hall/CRC, 2003.

[15] D. N. Clark and G. Misra, Curvature and similarity, Mich Math J 30 (1983), 361 - 367.

[16] On weighted shifts, curvature and similarity, J. London Math. Soc. 31 (1985), 357 - 368.

[17] M. J. Cowen and R. G. Douglas, Complex geometry and operator theory, Acta Math. 141 (1978), 187 - 261.

[18] _ On operators possessing an open set of eigenvalues, Memorial Conf. for Féjer-Riesz, Colloq. Math. Soc. J. Bolyai, 1980, $323-341$.

[19] _ On moduli for invariant subspaces, pp. 65-73, Birkhäuser Basel, Basel, 1982.

[20] _ Equivalence of Connections, Adv. Math. 56 (1985), 39 - 91.

[21] R. E. Curto and N. Salinas, Generalized Bergman kernels and the Cowen-Douglas theory, Amer J. Math. 106 (1984), 447 488.

[22] S. K. Donaldson, Anti self-dual Yang-Mills Connection over complex algebraic surfaces and stable vector bundles, Proc. London Math. Soc. 50 (1985), 1 - 26.

[23] R. G. Douglas, H.-K. Kwon, and S. Treil, Similarity of n-hypercontractions and backward Bergman shifts, J. London Math. Soc. 88 (2013), $237-648$.

[24] R. G. Douglas and G. Misra, Geometric invariants for resolutions of Hilbert modules, Operator Theory: Advances and Applications, Birkhauser, 1993, 83 - 112.

[25] __ Equivalence of quotient Hilbert modules, Proc. Indian Acad. Sc. (Math. Sc.) 113 (2003), 281 - 291.

[26] _ Quasi-free resolutions of Hilbert modules, Integral Equations and Operator Theory 47 (2003), 435 - 456.

[27] _ Equivalence of quotient modules - II, Trans. Amer. Math. Soc. 360 (2008), 2229 - 2264.

[28] R. G. Douglas, G. Misra, and C. Varughese, On quotient modules - the case of arbitrary multiplicity, J. Func. Anal. 174 (2000), $364-398$.

[29] R. G. Douglas and V. I. Paulsen, Hilbert modules over function algebras, Pitman research notes in mathematics, no. 217, Longman Scientific and Technical, 1989.

[30] R. G. Douglas, V. I. Paulsen, C.-H. Sah, and K. Yan, Algebraic reduction and rigidity for Hilbert modules, Amer. J. Math. 117 (1995), $75-92$. 
[31] J. Eschmeier and M. Putinar, Spectral decompositions and analytic sheaves, Oxford University Press, 1996.

[32] J. Eschmeier and J. Schmitt, Cowen-Douglas operators and dominating sets, J. Operator Theory 72 (2014), 277 - 290.

[33] S. Ghara, Decomposition of the tensor product of Hilbert modules via the jet construction and weakly homogeneous operators, $\mathrm{PhD}$ thesis, Indian Institute of Science, 2018.

[34] H. Grauert and R. Remmert, Coherent analytic sheaves, Springer-Verlag, Berlin, 1984.

[35] F. R. Harvey and H. B. Lawson, A theory of characteristic currents associated with a singular connection, Asterisque (1993), $1-268$.

[36] C. Jiang, Similarity Classification of Cowen-Douglas Operators, Canad. J. Math. 56 (2004), 742 - 775.

[37] C. Jiang and K. Ji, Similarity classification of holomorphic curves, Adv. Math. 215 (2007), 446 - 468.

[38] K. Ji, C. Jiang, D. K. Keshari, and G. Misra, Rigidity of the flag structure for a class of Cowen-Douglas operators, J. Func. Anal. 272 (2017), $2899-2932$.

[39] C. Jiang, K. Ji, and G. Misra, Classification of quasi-homogeneou holomorphic curves and operators in the Cowen-Douglas class, J. Func. Anal. 273 (2017), 2870 - 2915.

[40] C. Jiang and Z. Wang, Strongly irreducible operators on Hilbert space, Pitman Research Notes in Mathematics Series, vol. 389, Longman, Harlow, 1998.

[41] A. Kirillov, Elements of the theory of representations, Springer-Verlag, 1976.

[42] A. Korányi and G. Misra, Homogeneous operators on Hilbert spaces of holomorphic functions, J. Func. Anal. 254 (2008), $2419-2436$.

[43] _ A classification of homogeneous operators in the Cowen - Douglas class, Integral Equations and Operator Theory 63 (2009), 595 - 599.

[44] __ A classification of homogeneous operators in the Cowen-Douglas class, Adv. Math. 226 (2011), 5338 - 5360.

[45] _ Homogeneous Hermitian holomorphic vector bundles and the Cowen-Douglas class over bounded symmetric domains, C. R. Math. Acad. Sci. Paris 354 (2016), 291 - 295.

[46] Homogeneous hermitian holomorphic vector bundles and the Cowen-Douglas class over bounded symmetric domains, arXiv:1806.01955, 2018.

[47] G. Misra, Curvature and the backward shift operator, Proc. Amer. Math. Soc. 91 (1984), 105 - 107.

[48] _ Curvature inequalities and extremal properties of the bundle shifts, J. Operator Theory 11 (1984), 305 - 317.

[49] G. Misra and A. Pal, Contractivity, complete contractivity and curvature inequalities, arXiv:1410.7493 to appear, J. d'Analyse Mathematique (2018).

[50] G. Misra and Md. Ramiz Reza, Curvature inequalities and extremal operators, 2018.

[51] G. Misra and S. Shyam Roy, The curvature invariant for a class of homogeneous operators, Proc. London Math. Soc. 99 (2009), $557-584$.

[52] G. Misra and N. S. N. Sastry, Homogeneous tuples of operators and holomorphic discrete series representation of some classical groups, J. Operator Theory 24 (1990), 23 - 32.

[53] G. Misra and H. Upmeier, Homogeenous vector bundles and intertwining operators for symmetric domains, Adv. Math. 303 (2016), 1077 - 1121.

[54] L. Peng and G. Zhang, Tensor products of holomorphic representations and bilinear differential operators, J. Func. Anal. 210 (2004), $171-192$.

[55] Md. Ramiz Reza, Curvature inequalities for operators in the cowen-douglas class of a planar domain, Indiana Univ. Math. J. 67 (2018), 1255 - 1279.

[56] W. Rudin, Analytic functions of class $H_{p}$, Trans. Amer. Math. Soc. 78 (1955), 46 - 66.

[57] D. Sarason, The $H^{p}$ spaces of an annulus, Mem. Amer. Math. Soc. No. 56 (1965), 78.

[58] N. Suita, On a metric induced by analytic capacity. II, Kōdai Math. Sem. Rep. 27 (1976), 159 - 162.

[59] K. Wang, and G. Zhang, Curvature inequalities for operators of the Cowen-Douglas class, Israel J. Math. 222 (2017), 279 296.

[60] D. R. Wilkins, Homogeneous vector bundles and Cowen-Douglas operators, Intern. J. Math. 4 (1993), 503 - 520.

[61] D. V. Yakubovich, Dual piecewise analytic bundle shift models of linear operators, J. Func. Anal. 136 (1996), 294 - 330.

[62] K. Zhu, Operators in Cowen-Douglas classes, Illinois J. Math. 44 (2000), 767 - 783.

E-mail address: gmeisc.ac.in

Department of Mathematics, Indian Institute of Science, Bangalore 560012 